\title{
1 Foxc1 establishes enhancer accessibility for craniofacial cartilage
}

\section{2 differentiation}

3 Pengfei Xü, Haoze Vincent $\mathrm{Yu}^{\#}$, Kuo-Chang Tseng, Mackenzie Flath, Peter Fabian, Neil Segil

4 and J. Gage Crump*

6 Eli and Edythe Broad Center for Regenerative Medicine, Department of Stem Cell Biology and

7 Regenerative Medicine, Keck School of Medicine, University of Southern California, Los

8 Angeles, CA 90033, USA

10 *Correspondence: J. Gage Crump, gcrump@usc.edu, (323) 442-2693

11 \#These authors contributed equally

\section{Highlights}

14 - Dynamic chromatin accessibility across facial cartilage development

15 - Co-enrichment of Fox- and Sox-binding motifs in accessible regions

16 - Foxc1 establishes accessibility in a subset of facial cartilage enhancers

17 - Modular activity of Foxc1-dependent cartilage enhancers in zebrafish 


\section{Abstract}

19 The specification of cartilage requires Sox9, a transcription factor with broad roles for

20 organogenesis outside the skeletal system. How Sox9 gains selective access to cartilage-specific

21 cis-regulatory regions during skeletal development had remained unclear. By analyzing chromatin

22 accessibility during the differentiation of neural crest cells into chondrocytes of the zebrafish head,

23 we find that cartilage-associated chromatin accessibility is dynamically established. Cartilage-

24 associated regions that become accessible after neural crest migration are co-enriched for Sox9

25 and Fox transcription factor binding motifs. In zebrafish lacking Foxc1 paralogs, we find a global

26 decrease in chromatin accessibility in chondrocytes, consistent with a later loss of dorsal facial

27 cartilages. Zebrafish transgenesis assays confirm that many of these Foxc1-dependent elements

28 function as enhancers with region- and stage-specific activity in facial cartilages. We propose that

29 Foxc1-dependent chromatin accessibility helps directs the versatile Sox9 protein to a

30 chondrogenic program in the face.

31

\section{Introduction}


33 Cartilage is the first skeletal type to be specified in the vertebrate body, providing important

34 templates for later bone development and providing flexibility at joint surfaces and within the

nose, ear, ribs, and larynx. The transcription factor Sox9 is essential for chondrogenic

36 differentiation in all vertebrates examined (Lefebvre et al., 1997; Bi et al., 1999; Mori-Akiyama et

37 al., 2003; Yan et al., 2005), yet it also has widespread roles outside the skeletal system,

38 including in the reproductive system, kidney, liver, and skin (Jo et al., 2014). How Sox9 is

39 directed to a chondrogenic program in trunk mesoderm and cranial neural crest-derived cells

40 (CNCCs) has remained unclear. Sox9 is known to directly bind to a number of cis-regulatory

41 elements adjacent to chondrogenic genes, including Col2a1, Col10a1, and Acan (Lefebvre et

42 al., 1997; Dy et al., 2012; Askary et al., 2015; Ohba et al., 2015). However, it is not required for

43 chromatin accessibility at these same elements (Liu et al., 2018), suggesting other unknown

44 factors may first open chromatin at chondrogenic enhancers for later activation by Sox9.

46 Forkhead-domain (Fox) family transcription factors are excellent candidates for making

47 chondrogenic enhancers accessible for later Sox9 activation. In the endoderm lineage, 
al., 2002). A similar chromatin accessibility role for Foxd3 in the early neural crest lineage has

52 (Hong et al., 1999), loss of calvarial bone due to premature ossification (Rice et al., 2003;

53 Vivatbutsiri et al., 2008; Sun et al., 2013), syngnathia (Inman et al., 2013), and disruption of

54 endochondral bone maturation (Yoshida et al., 2015). In zebrafish, loss of both Foxc1 paralogs

55 (foxc1a and foxc1b) results in severe reductions of dorsal cartilages of the upper face, which are

preceded by reduced expression of several Sox9 targets, including col2a1a, acana, matn1, and

matn4 (Xu et al., 2018). Chromatin immunoprecipitation followed by deep sequencing (ChIP-

58 Seq) using a Sox9 antibody in dissected mouse rib and nose cartilage revealed enrichment of

59 Fox binding motifs within Sox9-bound cis-regulatory sequences near chondrogenic genes

60 (Ohba et al., 2015). Here, we use profiling of chromatin accessibility in wild-type and mutant

61 zebrafish facial cartilages and find that Foxc1 paralogs are required for accessibility and activity

62 of a number of cartilage enhancers. These findings support a model by which Foxc1 restricts

63 Sox9 activity to a chondrogenic program by establishing selective accessibility of cartilage 


\section{Results and Discussion}

\section{Chromatin accessibility landscape in chondrocytes of the zebrafish face}

67 In order to identify potential cis-regulatory elements important for facial cartilage development,

"micro" version of the assay for transposase accessible chromatin followed by next-generation 
81 1D; Supplementary file 1A). Sox9 ChIP-Seq of mouse chondrocytes had previously revealed

82 Nfat and Fox motifs as the second and third most co-enriched with Sox motifs (Ohba et al.,

83 2015), and the Nkx motif might reflect the role of Nkx3.2 in cartilage differentiation (Provot et al.,

84 2006). Consensus sequences for Sox, Fox, and Nfat motifs were highly similar between

85 zebrafish and mouse (Figure 1-figure supplement 1B), despite our zebrafish analysis focusing

86 on all accessible regions in facial chondrocytes and mouse analysis focusing on only Sox9-

87 bound regions in rib chondrocytes. These striking motif similarities indicate strong conservation

88 of the cartilage gene regulatory network between fish and mammals, and between the face and

89 rib, and strongly suggest that many of the identified chondrocyte-specific accessible regions in

90 zebrafish likely function as chondrocyte enhancers. In addition, Gene Ontology (GO) analysis of

91 the nearest genes to the chondrocyte-enriched elements revealed cartilage development as one

92 of the top 6 associated terms (Figure 1E). We also recovered terms for neural crest cell

93 migration and dorsal/ventral pattern formation, likely reflecting retention of enhancer

94 accessibility linked to the neural crest origins and later dorsoventral arch patterning of the

95 precursors of facial cartilage. 


\section{Progressive establishment of cartilage-associated chromatin accessibility}

98 We next investigated when cartilage-associated chromatin accessibility is established in relation

99 to CNCC development. CNCCs are first specified at $\sim 10.5 \mathrm{hpf}$ at the border of the neural keel,

100 finish their migration into the pharyngeal arches by $\sim 20 \mathrm{hpf}$, and then show the first histological

101 signs of cartilage development in the jaw at $\sim 52 \mathrm{hpf}$ (Schilling and Kimmel, 1997). CNCC-

102 derived arch ectomesenchyme cells can be uniquely identified by co-expression of fli1a:GFP

103 and sox10:Dsred transgenes at $36 \mathrm{hpf}$ and $48 \mathrm{hpf}$ (Askary et al., 2017), stages just prior to

104 cartilage differentiation (Figure 2A). We performed $\mu$ ATACseq on fli1a:GFP+; sox10:Dsred+

105 cells after FACS and analyzed chondrocyte-specific accessible elements from our $72 \mathrm{hpf}$

106 dataset for their accessibilities at 36 and $48 \mathrm{hpf}$ (Figure 2B). Of the 5,736 elements enriched in

107 chondrocytes at $72 \mathrm{hpf}$, only 6\% (356) had peak accessibility at $36 \mathrm{hpf}$ with no further increase

108 in accessibility by 48 hpf (“Group I”). In contrast, $48 \%(2,741)$ displayed increased accessibility

109 between 36 and 48 hpf (“Group II”), and 46\% (2,639) between 48 and 72 hpf (“Group III”). For

110 Group I, de novo motif enrichment revealed predicted binding sites for members of the Nfat,

111 Fox, Lhx, Nr2f, Meis and Pax families, and significant GO terms included neural crest migration,

112 cell migration in general, and dorsal/ventral pattern formation (Figure 2C,D). Combined with the 
113 known involvement of Foxd3 (Montero-Balaguer et al., 2006; Stewart et al., 2006), Lhx6/8

114 (Denaxa et al., 2009), Nr2f1/2/5 (Barske et al., 2018), Meis2 (Machon et al., 2015) and Pax9

115 (Nakatomi et al., 2010) in CNCC specification, migration, and dorsal-ventral arch patterning,

116 many Group I elements likely represent retention of cis-regulatory elements involved in the

117 earlier specification, migration, and regional patterning of CNCCs. Group II and Group III

118 elements share many common predicted transcription factor binding motifs, including Sox

119 dimer, Fox, Nfat, and Ap1 motifs previously described for mouse cartilage (Figure 2E,G;

120 Supplementary file 1B) (Ohba et al., 2015). An Nkx motif was recovered only for Group II ( $p=10^{-}$

$121{ }^{58}, 36 \%$ of targets), an Egr motif was enriched for Group II ( $p=10^{-65}, 55 \%$ of targets) versus

122 Group III ( $p=10^{-20}, 16 \%$ of targets), and a Tead motif was enriched for Group III ( $p=10^{-63}, 36 \%$ of

123 targets) versus Group II ( $p=10^{-20}, 3 \%$ of targets). GO analysis for linked genes also revealed

124 terms related to skeletal system development (Group II) and cartilage development (Group III),

125 as well as more general terms such as transcription and organism development (Figure $2 \mathrm{~F}, \mathrm{H}$ ).

126 We therefore conclude that the majority of chondrocyte-specific elements gain accessibility after

127 pharyngeal arch formation, and that transcription factor binding motifs change during cartilage

128 differentiation. For example, enrichment of Nkx motifs in Group II elements might reflect the role 
129 of Nkx3.2 in limiting chondrocyte maturation (Provot et al., 2006) and promoting joint formation

130 (Miller et al., 2003), while the preferential enrichment of the Tead motif, which is linked to

131 growth-associated Hippo signaling (Ota and Sasaki, 2008), in Group III elements might reflect

132 the later proliferative expansion of chondrocytes.

134 Requirement of Foxc1 for chromatin accessibility at a subset of cartilage elements

135 We had previously found that Foxc1 genes are essential for cartilage development in the upper

136 face (Xu et al., 2018), and both our $\mu$ ATACseq analysis of zebrafish chondrocytes and

137 published Sox9 ChIP-seq analysis in mouse (Ohba et al., 2015) reveals co-enrichment of Sox

138 and Fox motifs in accessible regions near known cartilage genes. In zebrafish Foxc1 (foxc1a-/-;

139 foxc1b-/-) mutants, cartilages of the upper/dorsal face fail to develop (Xu et al., 2018). In order

140 to isolate the dorsal CNCC precursors affected in Foxc1 mutants, we used a pou3f3b:Gal4;

141 UAS:n/sGFP (pou3f3b>GFP) dorsal CNCC transgenic line (\#Barske et al., 2020, PNAS, in

142 press) along with the pan-CNCC sox10:Dsred transgenic line. Whereas pou3f3b>GFP+;

143 sox10:Dsred+ CNCCs were present in Foxc1 mutant pharyngeal arches at 48 hpf, many fewer

144 differentiated into chondrocytes by 6 days post-fertilization (dpf) compared to sibling controls 
145 (Figure 3A-D). We therefore performed $\mu$ ATACseq on pou3f3b>GFP+; sox10:Dsred+ cells after

146 FACS from Foxc1 mutants and controls at 36 and $48 \mathrm{hpf}$. A comparison of the 15,781

147 accessible regions in dorsal CNCCs (pou3f3b>GFP+; sox10:Dsred+) and pan-CNCCs

148 (fli1a:GFP+; sox10:Dsred+) at $36 \mathrm{hpf}$ revealed $79 \%$ with similar accessibility, $11 \%$ with greater

149 accessibility in dorsal CNCCs, and 10\% with greater accessibility in pan-CNCCs (Figure 3-figure

150 supplement $1 \mathrm{~A}$ ). Of 22,323 elements at $48 \mathrm{hpf}, 72 \%$ were similarly accessible, $10 \%$ more

151 accessible in dorsal CNCCs, and 18\% more accessible in pan-CNCCs (Figure 3-figure

152 supplement 1B). A comparison of the 5,736 regions with specific accessibility in cartilage at 72

153 hpf revealed high correlation between dorsal- and pan-CNCCs at $36 \mathrm{hpf}$, with 96\% displaying

154 similar accessibility ( $r=0.92$, Figure 3-figure supplement 2A,C). By 48 hpf, however, we

155 observed notable differences between accessibility of cartilage-specific elements, with $43 \%$

156 displaying greater accessibility in pan-CNCCs and only $0.2 \%$ displaying greater accessibility in

157 dorsal CNCCs ( $r=0.71$, Figure 3-figure supplement 2B,D). The decreased accessibility of

158 cartilage-specific elements in dorsal CNCCs at $48 \mathrm{hpf}$ supports previous studies that dorsal

159 chondrocytes develop later than other chondrocytes in the zebrafish face (Schilling and Kimmel,

160 1997; Barske et al., 2016). 
161 Analysis of cartilage-associated elements in Foxc1 mutants revealed that $10 \%(120 / 1,221)$ of

162 elements that have established peak accessibility by $36 \mathrm{hpf}$ (i.e. Group I) and 41\% $(636 / 1,556)$

163 of elements that increase accessibility between 36 and 48 hpf (i.e. Group II) had reduced

164 accessibility in Foxc1 mutants (Figure 3E). De novo motif analysis of Foxc1-dependent and

165 Foxc1-independent elements in Group I showed enrichment of Sox $\left(p=10^{-12}, p=10^{-76}\right)$, Tead

$166\left(p=10^{-14}, p=10^{-20}\right) \operatorname{Mtf}\left(p=10^{-12}, p=10^{-22}\right)$ and Ets $\left(p=10^{-12}, p=10^{-61}\right)$ motifs in both (Figure 3F,

167 Supplementary file 1C). Whereas Fox motifs were uncovered in both, a closer analysis revealed

168 enrichment of Foxa2 and Foxd3 motifs only in Foxc1-dependent elements $\left(p=10^{-45}, 38 \%\right.$ of

169 targets; $p=10^{-16}, 18 \%$ of targets), and a Foxo1 motif only in Foxc1-independent elements $\left(p=10^{-}\right.$

$170 \quad 42,49 \%$ of targets). Similarly in Group II elements, a Foxa1 motif was enriched only in Foxc1-

171 dependent elements ( $p=10^{-59}, 38 \%$ of targets), and Foxh1 and Foxp1 motifs only in Foxc1-

172 independent elements $\left(p=10^{-22}, 22 \%\right.$ of targets; $p=10^{-19}, 7 \%$ of targets) (Figure $3 G$,

173 Supplementary file 1C). Although Foxc1 motifs were not in the database used for motif

174 predictions, the selective presence of Foxa1/2 motifs in Foxc1-dependent elements is consistent

175 with previous reports that the consensus sequence for Foxc1-bound peaks is nearly identical to

176 Foxa1/2 motifs (Wang et al., 2016). Sox motifs were similarly enriched in both Group I and 
177 Group II Foxc1-dependent and -independent elements, and Nfat and Zfx motifs in Group II

178 Foxc1-dependent and -independent elements. An Insm ( $p=10^{-39}, 30 \%$ of targets $)$ motif was

179 uncovered only in Foxc1-independent Group I elements, and an Nkx motif $\left(p=10^{-35}, 52 \%\right.$ of

180 targets) only in Foxc1-independent Group II elements. These findings suggest that Foxc1-

181 dependent and -independent cartilage elements are commonly bound by Sox9 but likely differ in

182 co-binding by Foxc1 and additional co-factors. For example, the presence of the Nkx motif only

183 in Foxc1-independent elements suggests that it could be an alternative co-factor for Sox9, in

184 line with the known roles for Nkx3.2 in chondrocyte biology (Provot et al., 2006).

185

186 Validation of Foxc1-dependent cartilage enhancers in zebrafish transgenesis assays

187 To verify whether Foxc1-dependent cartilage elements identified by $\mu$ ATACseq are

188 chondrogenic enhancers, we tested the ability of individual elements in combination with an E1b

189 minimal promoter to drive cartilage expression of green fluorescent protein (GFP) in zebrafish

190 transgenic assays (Figure 4-figure supplement 1A). We tested 22 Foxc1-dependent elements

191 near 15 different genes, which included elements linked to genes with known cartilage function

192 (ucmab, matn4, matn1, lect1, epyc, col9a1a, col9a3, sox10, acana, foxa3, mia) and others with 
193 unknown cartilage function (si:dkey33i1l.4, gas1b, lefty2, slc35d1a) (Figure 4A; Figure 4-figure

194 supplement 1; Supplementary file 2). We observed that $59 \%$ (13/22) of elements drove GFP

195 expression in facial chondrocytes at $6 \mathrm{dpf}$. These included intronic elements within sox10, lect1,

196 col9a3, col9a1a and slc35d1a; distal 5' elements near ucmab, epyc, mia, acana, and matn4; a

197 distal 3' element near gas1b; and a promoter-associated element for lect1. All tested elements

198 are from Group II, i.e. increasing accessibility between 36 and 48 hpf, and we confirmed

199 cartilage expression in independent stable transgenic lines in 9 cases (Figure 4B; Figure 4-

200 figure supplement 1; Supplementary file 2). Whereas an element in the first intron of sox10

201 drove uniform cartilage-specific expression, most elements drove expression in specific sub-

202 regions or differentiation stages of facial chondrocytes. Whereas a distal 5' element of ucmab

203 drove expression in chondrocytes of multiple joints in the zebrafish head, an intronic element of

204 lect1 drove chondrocyte expression only in the jaw joint and hyomandibular-otic connection, a

205 promoter-associated element of lect1 drove expression more strongly in the hyoid joint and

206 hyomandibular-symplectic connection (though also more broadly in chondrocytes), and a distal

2075 ' element of acana drove restricted chondrocyte expression at the hyoid joint. Reciprocally,

208 elements associated with col9a3, epyc, mia, and col9a1a were expressed in chondrocytes but 
209 generally excluded from joint regions (particularly apparent at the hyoid joint and

210 hyomandibular-symplectic connection). Further, we found that three enhancer transgenes with

211 diverse expression patterns (broad sox10, joint-restricted ucmab, and joint-excluded epyc

212 enhancers) all displayed reduced activity specifically in the dorsal cartilage regions affected in

213 Foxc1 mutants (Figure 4C). We also tested four Foxc1-independent elements and found that

214 two drove cartilage expression (distal 5' elements of gas1b and matn4), one drove ligament

215 expression (distal 5' element of sparc), and one had no activity (promoter-associated element of

216 sox10) (Figure 4-figure supplement 1). Thus, the majority of accessible elements tested had

217 chondrocyte activity, and Foxc1-dependent and -independent elements appear equally capable

218 of driving cartilage expression. Moreover, the activity of different enhancers in diverse locations

219 and stages of chondrocyte maturation indicates that global cartilage expression patterns are

220 achieved in part through the summation of enhancers with more restricted activity.

\section{Conclusion}

223 Our findings indicate that Foxc1 function is required for the accessibility of close to half of

224 chondrocyte enhancers in the zebrafish face. Given expression and function of Foxc1 in diverse 
225 cartilages (e.g. limb, rib, tracheal) in mouse, it seems likely that Foxc1 has a similar function in

226 chondrocyte enhancer accessibility throughout the body. It is unclear why only a minority of

227 enhancers appears to require Foxc1 activity. It is possible that other members of the Fox family

228 compensate, such as Foxf1/2 in the facial midline (Xu et al., 2018) and Foxa2/3 during later

229 hypertrophic maturation (Ionescu et al., 2012). Alternatively, there may be other co-factors that

230 cooperate with Sox9 for chondrocyte enhancer accessibility and activation, such as Nkx3.2. We

231 did not detect any obvious differences in the types of enhancer-proximal genes or the patterns

232 and stages associated with Foxc1-dependent versus -independent enhancer activity. Further

233 work will also be needed to understand the mechanism by which Foxc1 promotes chondrocyte

234 enhancer accessibility. Foxc1 lacks a chromatin modifying domain (Yoshida et al., 2015) and

235 therefore would need to interact with a co-factor to directly open chromatin. Foxc1 could also act

236 to maintain open chromatin, as shown for Foxa1 in the liver (Reizel et al., 2020). Given that both

237 Foxc1 and Sox9 have expression in many tissues outside the skeletal system, it will also be

238 important to determine whether additional factors help to further restrict their activity to

239 chondrocyte enhancers within skeletogenic mesenchyme. 


\section{Methods}

\section{Zebrafish lines}

243 The Institutional Animal Care and Use Committee of the University of Southern California

244 approved all experiments on zebrafish (Danio rerio) (Protocol \#10885). Existing mutant and

245 transgenic lines used in this study include foxc1 $a^{e / 542}$ and foxc $1 b^{e / 620}$ (Xu et al., 2018);

$246 \mathrm{Tg}(\text { sox10:Dsred) })^{\mathrm{el110}}$ and Tg(fli1a:EGFP) ${ }^{\mathrm{y} 1}$ (Askary et al., 2017); Tg(col2a1aBAC:GFP) (Paul et

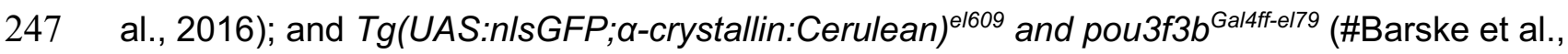

248 2020, PNAS, in press). For enhancer transgenic lines, we synthesized accessible elements with

249 flanking attB4 and attB1 sequences using IDT gBlocks and cloned these into pDONR-P4-P1R

250 using the Gateway Tol2kit (Invitrogen) to create p5E enhancer constructs (Kwan et al., 2007).

251 We then combined p5E constructs with pME-E1b-GFP, p3E-polyA, and pDestTol2AB2 using LR

252 clonase. Final DNA constructs were microinjected with transposase RNA (30 ng/ $\mu \mathrm{l}$ each) into 1-

253 cell stage zebrafish embryos. In most cases, multiple independent stable alleles per construct

254 were analyzed in the F1 generation (Supplementary file 2). 
257 Transgenic embryos were imaged with a Zeiss LSM800 confocal microscope. Maximum

258 intensity projections are shown for stable transgenic lines and single representative sections are

259 shown for injected embryos. Image levels were modified consistently across samples in Adobe

260 Photoshop CS6.

263 Wild-type embryos double-positive for fli1a:EGFP and sox10:Dsred (36 hpf and $48 \mathrm{hpf}$ ), or

264 col2a1a:GFP and sox10:Dserd (72 hpf), were sorted under a fluorescent dissecting microscope

265 (Leica M165FC) before dissociation. For Foxc1 mutant analysis, we performed incrosses of

266 pou3f3b:Gal4+/-; UAS:n/s-GFP+/-; sox10:Dsred+/-; foxc1a+/-; foxc1b+/- fish and then selected

267 for GFP+/Dsred+ embryos on a fluorescent dissecting microscope. Genotyping was then

268 performed on tail lysates collected from individual embryos at $27 \mathrm{hpf}$. We then pooled foxc1a-/-;

269 foxc1b-/- double mutants and separate sibling controls (foxc1a+/-; foxc $1 b+/+$ and foxc1a+/+;

270 foxc $1 b+/+$ embryos) for FACS. To facilitate embryo collection at the $36 \mathrm{hpf}$ time point, embryos

271 were moved at $27 \mathrm{hpf}$ to an incubator set at $22^{\circ} \mathrm{C}$ to delay their development such that they

272 reached $36 \mathrm{hpf}$ the following morning. Cell dissociation and FACS were performed as previously 
273 described (Askary et al., 2017). Around 5000 cells of each sample were centrifuged at $500 \mathrm{~g}$ for

$27420 \mathrm{~m}$ at $4{ }^{\circ} \mathrm{C}$, and the pellet was suspended with $20 \mu \mathrm{L}$ of lysis buffer $(10 \mathrm{mM}$ Tris $\mathrm{HCl}(\mathrm{pH} 7.4)$,

$2755 \mathrm{mM} \mathrm{MgCl}_{2}, 10 \% \mathrm{DMF}, 0.2 \% \mathrm{~N}-\mathrm{P} 40$ ) by pipetting 6-10 times to release the nuclei without

276 purification. The cell lysate was then mixed with $30 \mu \mathrm{L}$ reaction buffer $(10 \mathrm{mM} \mathrm{Tris} \mathrm{HCl}(\mathrm{pH} 7.4)$,

$2775 \mathrm{mM} \mathrm{MgCl}_{2}, 10 \% \mathrm{DMF}$, and homemade Tn5 Transposase) by vortexing for $5 \mathrm{~s}$. The reaction

278 was incubated at $37^{\circ} \mathrm{C}$ for 20 minutes, followed by DNA purification using a Qiagen MiniElute

279 kit. Purified DNA fragments were used to conduct $\mu$ ATACseq libraries as previously described

280 (Buenrostro et al., 2013) and sequenced using the NextSeq 500 platform (Illumina) with a

281 minimum of 50 million paired-reads/sample.

282

283 Data analysis and statistics

284 The Encode analysis pipeline (https://github.com/ENCODE-DCC/chip-seq-pipeline) for

285 ATACseq was used with small modifications. The raw reads were trimmed to 37 bp and aligned

286 to zebrafish GRCz10 genome assembly by STAR aligner (Dobin et al. 2013). PCR duplicates,

287 and the reads that aligned to "blacklist regions" (Amemiya et al. 2019) were removed, and then

288 peaks were called by Model-based analysis of ChIP-Seq (MACS2) (Zhang et al. 2008) with 
$p=0.01$ cutoff and disabled dynamic lambda option (--nolambda) for individual replicates. Peaks

290 from individual replicates were further filtered by IDR $<0.01$, and the overlapping peaks between

291 replicates were used. Bigwig files were generated from dup-removed bam files with bedtools

292 and bedgraphtobigwig, and normalization was based on total read numbers. Heatmaps were

293 generated with DeepTools (Ramírez et al. 2016) based on the normalized bigwig signal files.

294 Individual genomic loci were examined by IGV (Broad Institute). Differential analyses of

295 HATACseq data was done with DESeq2 (Love et al. 2014). HOMER (Heinz et al. 2010) was

296 used to identify de novo motifs. David 6.8 GO analysis was performed on the web interface

297 (https://david.ncifcrf.gov/) based on nearest neighbor genes for all differentially accessible

298 elements. Pearson correlation was calculated using cor() function in R.

300 Acknowledgements

301 We thank Jeffrey Boyd at the USC Stem Cell Flow Cytometry Core for FACS, David Ruble at

302 the CHLA Sequencing Core, the high-performance computing core at USC, and Megan

303 Matsutani, Jennifer DeKoeyer Crump, and Mathi Thiruppathy for fish care. We dedicate this

304 work to the late Bartosz Balczerski whose passion as a postdoc inspired this project. 


\section{Author Contributions}

306 P.X. and J.G.C. conceived of experiments. P.X., M.F, and P.F. conducted zebrafish

307 experiments. P.X. and H.V.Y. performed $\mu$ ATACseq experiments, and with K.-C.T. conducted

308 bioinformatics analysis. N.S. and J.G.C. obtained funding and oversaw project. P.X. and J.G.C.

309 wrote the manuscript.

310

311 Competing interests

312 The authors declare no competing interests.

314 Materials \& Correspondence

315 Requests for material should be directed to J. Gage Crump (gcrump@usc.edu).

316 Data availability

317 MATACseq data can be accessed at GEO with accession number GSE157575:

318 visit https://www.ncbi.nlm.nih.gov/geo/query/acc.cgi?acc=GSE157575 ;!!LIr3w8kk Xxm!4o2V

319 xflwp2fIZI1FVkwGRA5Q9CcHhvfeKfQYZftKgRy6JUHBaaAPO2btB-Xgs0o\$ and enter token

320 gzcxqyourvmdnuj into the box. 


\section{References}

Askary, A., Xu, P., Barske, L., Bay, M., Bump, P., Balczerski, B., Bonaguidi, M. A. and Crump, J. G. (2017). Genome-wide analysis of facial skeletal regionalization in zebrafish. Development 144, 2994-3005.

Askary, A., Mork, L., Paul, S., He, X., Izuhara, A. K., Gopalakrishnan, S., Ichida, J. K., McMahon, A. P., Dabizljevic, S., Dale, R. et al. (2015). Iroquois Proteins Promote Skeletal Joint Formation by Maintaining Chondrocytes in an Immature State. Dev Cell 35, 358-365.

Barske, L., Rataud, P., Behizad, K., Del Rio, L., Cox, S. G. and Crump, J. G. (2018). Essential Role of Nr2f Nuclear Receptors in Patterning the Vertebrate Upper Jaw. Dev Cell 44, 337-347 e335.

Barske, L., Askary, A., Zuniga, E., Balczerski, B., Bump, P., Nichols, J. T. and Crump, J. G. (2016). Competition between Jagged-Notch and Endothelin1 Signaling Selectively Restricts Cartilage Formation in the Zebrafish Upper Face. PLoS Genet 12, e1005967.

Bi, W., Deng, J. M., Zhang, Z., Behringer, R. R. and de Crombrugghe, B. (1999). Sox9 is required for cartilage formation. Nat Genet 22, 85-89.

Buenrostro, J. D., Giresi, P. G., Zaba, L. C., Chang, H. Y. and Greenleaf, W. J. (2013). Transposition of native chromatin for fast and sensitive epigenomic profiling of open chromatin, DNA-binding proteins and nucleosome position. Nat Methods 10, 1213-1218.

Cirillo, L. A., Lin, F. R., Cuesta, I., Friedman, D., Jarnik, M. and Zaret, K. S. (2002). Opening of compacted chromatin by early developmental transcription factors HNF3 (FoxA) and GATA-4. Mol Cell 9, 279-289.

Denaxa, M., Sharpe, P. T. and Pachnis, V. (2009). The LIM homeodomain transcription factors Lhx6 and Lhx7 are key regulators of mammalian dentition. Dev Biol 333, 324-336.

Dy, P., Wang, W., Bhattaram, P., Wang, Q., Wang, L., Ballock, R. T. and Lefebvre, V. (2012). Sox9 directs hypertrophic maturation and blocks osteoblast differentiation of growth plate chondrocytes. Dev Cell 22, 597-609.

Hong, H. K., Lass, J. H. and Chakravarti, A. (1999). Pleiotropic skeletal and ocular phenotypes of the mouse mutation congenital hydrocephalus (ch/Mf1) arise from a winged helix/forkhead transcriptionfactor gene. Hum Mol Genet 8, 625-637. 
Inman, K. E., Purcell, P., Kume, T. and Trainor, P. A. (2013). Interaction between Foxc1 and Fgf8 during mammalian jaw patterning and in the pathogenesis of syngnathia. PLoS Genet 9, e1003949.

lonescu, A., Kozhemyakina, E., Nicolae, C., Kaestner, K. H., Olsen, B. R. and Lassar, A. B. (2012). FoxA family members are crucial regulators of the hypertrophic chondrocyte differentiation program. Dev Cell 22, 927-939.

Jo, A., Denduluri, S., Zhang, B., Wang, Z., Yin, L., Yan, Z., Kang, R., Shi, L. L., Mok, J., Lee, M. J. et al. (2014). The versatile functions of Sox9 in development, stem cells, and human diseases. Genes Dis 1, 149-161.

Kume, T., Deng, K. Y., Winfrey, V., Gould, D. B., Walter, M. A. and Hogan, B. L. (1998). The forkhead/winged helix gene Mf1 is disrupted in the pleiotropic mouse mutation congenital hydrocephalus. Cell 93, 985-996.

Kwan, K. M., Fujimoto, E., Grabher, C., Mangum, B. D., Hardy, M. E., Campbell, D. S., Parant, J. M., Yost, H. J., Kanki, J. P. and Chien, C. B. (2007). The Tol2kit: a multisite gateway-based construction kit for Tol2 transposon transgenesis constructs. Dev Dyn 236, 3088-3099.

Lefebvre, V., Huang, W., Harley, V. R., Goodfellow, P. N. and de Crombrugghe, B. (1997). SOX9 is a potent activator of the chondrocyte-specific enhancer of the pro alpha1(II) collagen gene. Mol Cell Biol 17, 2336-2346.

Liu, C. F., Angelozzi, M., Haseeb, A. and Lefebvre, V. (2018). SOX9 is dispensable for the initiation of epigenetic remodeling and the activation of marker genes at the onset of chondrogenesis. Development 145.

Lukoseviciute, M., Gavriouchkina, D., Williams, R. M., Hochgreb-Hagele, T., Senanayake, U., Chong-Morrison, V., Thongjuea, S., Repapi, E., Mead, A. and Sauka-Spengler, T. (2018). From Pioneer to Repressor: Bimodal foxd3 Activity Dynamically Remodels Neural Crest Regulatory Landscape In Vivo. Dev Cell 47, 608-628 e606.

Machon, O., Masek, J., Machonova, O., Krauss, S. and Kozmik, Z. (2015). Meis2 is essential for cranial and cardiac neural crest development. BMC Dev Biol 15, 40.

Miller, C. T., Yelon, D., Stainier, D. Y. and Kimmel, C. B. (2003). Two endothelin 1 effectors, hand2 and bapx1, pattern ventral pharyngeal cartilage and the jaw joint. Development 130, 1353-1365. 
Montero-Balaguer, M., Lang, M. R., Sachdev, S. W., Knappmeyer, C., Stewart, R. A., De La Guardia, A., Hatzopoulos, A. K. and Knapik, E. W. (2006). The mother superior mutation ablates foxd3 activity in neural crest progenitor cells and depletes neural crest derivatives in zebrafish. Dev Dyn 235, 3199-3212.

Mori-Akiyama, Y., Akiyama, H., Rowitch, D. H. and de Crombrugghe, B. (2003). Sox9 is required for determination of the chondrogenic cell lineage in the cranial neural crest. Proc Natl Acad Sci U S A 100, 9360-9365.

Nakatomi, M., Wang, X. P., Key, D., Lund, J. J., Turbe-Doan, A., Kist, R., Aw, A., Chen, Y.,

Ohba, S., He, X., Hojo, H. and McMahon, A. P. (2015). Distinct Transcriptional Programs Underlie Sox9 Regulation of the Mammalian Chondrocyte. Cell Rep 12, 229-243.

Ota, M. and Sasaki, H. (2008). Mammalian Tead proteins regulate cell proliferation and contact inhibition as transcriptional mediators of Hippo signaling. Development 135, 4059-4069.

Paul, S., Schindler, S., Giovannone, D., de Millo Terrazzani, A., Mariani, F. V. and Crump, J. G. (2016). Ihha induces hybrid cartilage-bone cells during zebrafish jawbone regeneration. Development 143, 2066-2076.

Provot, S., Kempf, H., Murtaugh, L. C., Chung, U. I., Kim, D. W., Chyung, J., Kronenberg, H. M. and Lassar, A. B. (2006). Nkx3.2/Bapx1 acts as a negative regulator of chondrocyte maturation. Development 133, 651-662.

Reizel, Y., Morgan, A., Gao, L., Lan, Y., Manduchi, E., Waite, E. L., Wang, A. W., Wells, A. and Kaestner, K. H. (2020). Collapse of the hepatic gene regulatory network in the absence of FoxA factors. Genes Dev 34, 1039-1050.

Retting, K. N., Song, B., Yoon, B. S. and Lyons, K. M. (2009). BMP canonical Smad signaling through Smad1 and Smad5 is required for endochondral bone formation. Development 136, 1093-1104.

Rice, R., Rice, D. P., Olsen, B. R. and Thesleff, I. (2003). Progression of calvarial bone development requires Foxc1 regulation of Msx2 and Alx4. Dev Biol 262, 75-87.

Schilling, T. F. and Kimmel, C. B. (1997). Musculoskeletal patterning in the pharyngeal segments of the zebrafish embryo. Development 124, 2945-2960. 
411 Stewart, R. A., Arduini, B. L., Berghmans, S., George, R. E., Kanki, J. P., Henion, P. D. and

412 Look, A. T. (2006). Zebrafish foxd3 is selectively required for neural crest specification,

413 migration and survival. Dev Biol 292, 174-188.

414 Sun, J., Ishii, M., Ting, M. C. and Maxson, R. (2013). Foxc1 controls the growth of the murine

415 frontal bone rudiment by direct regulation of a Bmp response threshold of Msx2.

416 Development 140, 1034-1044.

417 Vivatbutsiri, P., Ichinose, S., Hytonen, M., Sainio, K., Eto, K. and Iseki, S. (2008). Impaired

418 meningeal development in association with apical expansion of calvarial bone osteogenesis

419 in the Foxc1 mutant. J Anat 212, 603-611.

420 Wang, L., Siegenthaler, J. A., Dowell, R. D. and Yi, R. (2016). Foxc1 reinforces quiescence in 421 self-renewing hair follicle stem cells. Science 351, 613-617.

422 Xu, P., Balczerski, B., Ciozda, A., Louie, K., Oralova, V., Huysseune, A. and Crump, J. G.

423 (2018). Fox proteins are modular competency factors for facial cartilage and tooth specification. Development 145.

425 Yan, Y. L., Willoughby, J., Liu, D., Crump, J. G., Wilson, C., Miller, C. T., Singer, A., 426 Kimmel, C., Westerfield, M. and Postlethwait, J. H. (2005). A pair of Sox: distinct and overlapping functions of zebrafish sox 9 co-orthologs in craniofacial and pectoral fin development. Development 132, 1069-1083.

Yoshida, M., Hata, K., Takashima, R., Ono, K., Nakamura, E., Takahata, Y., Murakami, T., 431 Foxc1 is necessary for Ihh-Gli2-regulated endochondral ossification. Nat Commun 6, 6653. 

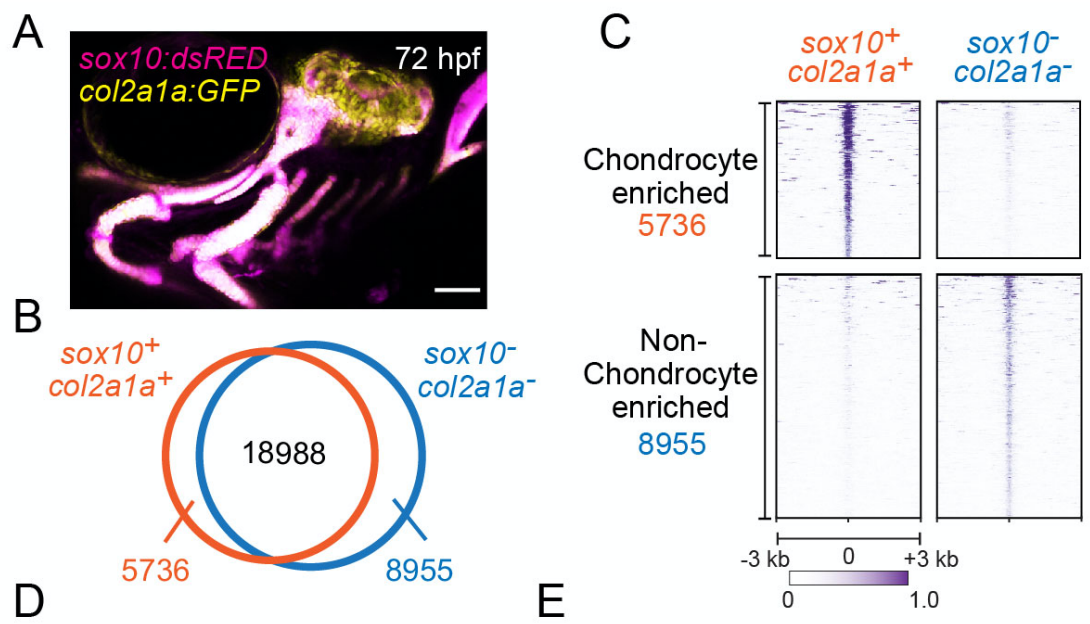

\begin{tabular}{|c|c|c|}
\hline TF family & Motif & $P$ value \\
\hline Sox & वर्टा होل & $1 e-206$ \\
\hline Fox & IG|TT & $1 e-107$ \\
\hline Nfat & 全 & $1 e-88$ \\
\hline Zfx & A\&AGGeses & $1 \mathrm{e}-71$ \\
\hline Nkx & $=I A_{\Delta} I_{3}$ & $1 e-53$ \\
\hline
\end{tabular}

\begin{tabular}{lc}
\multicolumn{2}{c}{ Gene Ontology } \\
GO Term & P value \\
Regulation of transcription & $1.5 \mathrm{e}-18$ \\
Multicellular organism development & $2.0 \mathrm{e}-14$ \\
Transcription & $4.2 \mathrm{e}-12$ \\
Dorsal/ventral pattern formation & $1.6 \mathrm{e}-10$ \\
Neural crest cell migration & $1.0 \mathrm{e}-9$ \\
Cartilage development & $9.0 \mathrm{e}-9$
\end{tabular}

Figure 1. Chromatin accessibility landscape of facial chondrocytes

433 (A) Confocal image of facial cartilages expressing col2a1a:GFP and sox10:Dsred at $72 \mathrm{hpf}$.

434 Lateral view with anterior to left. Scale bar $=100 \mu \mathrm{m} .(\mathrm{B})$ Venn diagram indicating distal

435 elements with open chromatin accessibility in col2a1a:GFP+; sox10:Dsred+versus

436 col2a1a:GFP-; sox10:Dsred-cells. (C) Peak intensity plots (Heatmap) of $\mu$ ATACseq show

437 differentially enriched open chromatin regions in double-positive versus double-negative cells.

438 (D) The top 5 transcription factor (TF) motifs recovered from the top $2000 \mu$ ATACseq peaks

439 enriched in chondrocytes (after removing redundant motifs). (E) GO analysis of nearest

440 neighbor genes of $\mu$ ATACseq peaks enriched in chondrocytes. 
bioRxiv preprint doi: https://doi.org/10.1101/2020 10.15.340703; this version posted October 15,2020 . The copyright holder for this preprint (which was not certified by peer review) is the author/funder, who has granted bioRxiv a license to display the preprint in perpetuity. It is made available under aCC-BY 4.0 International license.

A
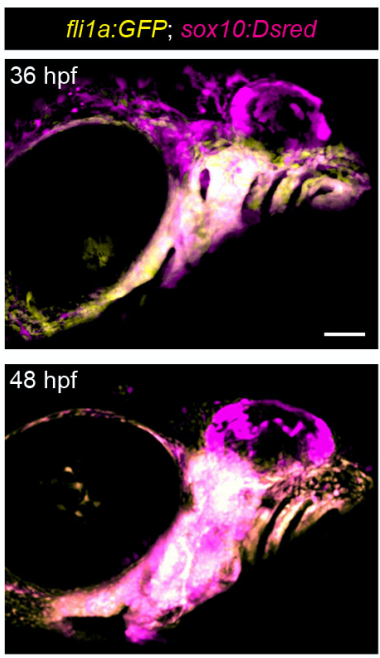

B

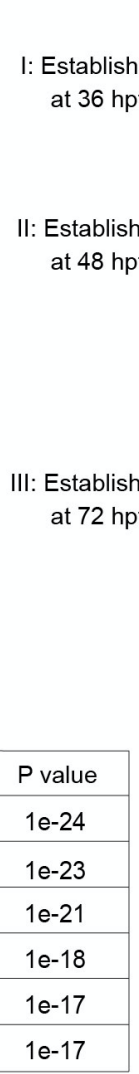

$\mathrm{E}$

\begin{tabular}{|c|c|c|c|}
\hline \multirow[t]{3}{*}{ C } & Motif & Best Match TFs & $P$ value \\
\hline & 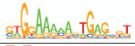 & Nfat & $1 e-24$ \\
\hline & cद्वा & Foxa2 & $1 e-23$ \\
\hline \multirow{4}{*}{ Group I } & cTAATAAGs: & Lhx & $1 e-21$ \\
\hline & 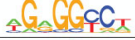 & $\mathrm{Nr} 2 \mathrm{f}$ & $1 e-18$ \\
\hline & 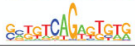 & Meis & $1 e-17$ \\
\hline & 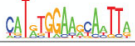 & Pax & $1 e-17$ \\
\hline
\end{tabular}

\begin{tabular}{|c|c|c|}
\hline Motif & Best Match TFs & $P$ value \\
\hline Adlese axd & Sox(Dimer) & $1 e-445$ \\
\hline IA \& AAACA & Foxa1 & $1 e-106$ \\
\hline TGGAAAAs & Nfat & $1 e-91$ \\
\hline E. CAClCAsarac & Egr & $1 \mathrm{e}-65$ \\
\hline AlsteAcast & Ap1 & $1 e-60$ \\
\hline CโCACA & Nkx & $1 e-58$ \\
\hline
\end{tabular}

G

\begin{tabular}{|c|c|c|c|}
\hline & Motif & Best Match TFs & $P$ value \\
\hline & $\therefore$ s TuAAAA. & Nfat & $1 e-155$ \\
\hline & $I=8 \times A A A C A$ & Foxa1 & $1 e-110$ \\
\hline \multirow[t]{4}{*}{ Group III } & Aatchese - & Sox(Dimer) & $1 e-73$ \\
\hline & THATCA. & Ap1 & $1 e-72$ \\
\hline & $A C A C A I T C C_{A C}$ & Tead & $1 e-63$ \\
\hline & $\triangle A A C G C B C E I T$ & Irf & $1 e-54$ \\
\hline
\end{tabular}

D

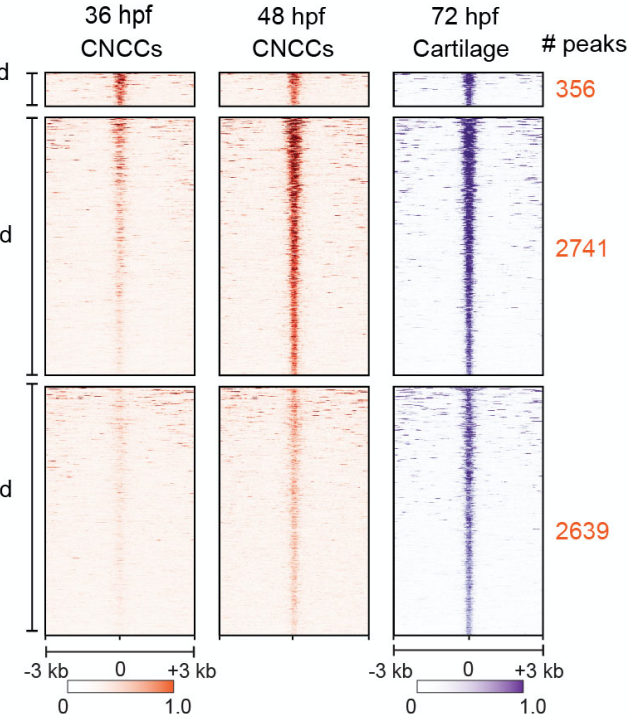

$\mathrm{F}$

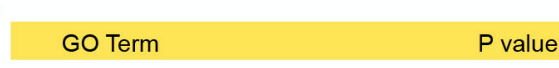

Transcription $\quad 9.6 \mathrm{e}-8$

Regulation of transcription $\quad 1.8 \mathrm{e}-7$

Cell differentiation $\quad 4.4 \mathrm{e}-7$

Skeletal system development $\quad 3.0 e-6$

Vasculature development $\quad 4.1 \mathrm{e}-6$

Multicellular organism development $\quad 5.0 \mathrm{e}-6$

$\mathrm{H}$

\begin{tabular}{lc}
\multicolumn{1}{c}{ GO Term } & P value \\
Regulation of transcription & $1.2 e-7$ \\
Dorsal/ventral pattern formation & $2.3 e-6$ \\
Cartilage development & $6.9 e-6$ \\
Transcription & $1.6 e-5$ \\
Retinal ganglion cell axon guidance & $1.3 e-4$ \\
Multicellular organism development & $1.6 e-4$
\end{tabular}


443 (A) Confocal images of CNCCs expressing fli1a:GFP and sox10:Dsred at 36 and 48 hpf. Lateral

444 view with anterior to left. Scale bar $=100 \mu \mathrm{m}$. (B) Peak intensity plots of cartilage-accessible

445 distal elements shown for chondrocytes at $72 \mathrm{hpf}$ and CNCCs at 36 and $48 \mathrm{hpf}$. Chondrocyte

446 accessible elements are pooled into three categories based on dynamics of chromatin

447 accessibility across stages. (C, E, G) De novo motif enrichment recovered by Homer analysis

448 among the three categories. Top 6 motifs are shown with associated $P$ values after removing

449 redundant motifs. (D, F, G) GO term analysis among the three categories. 

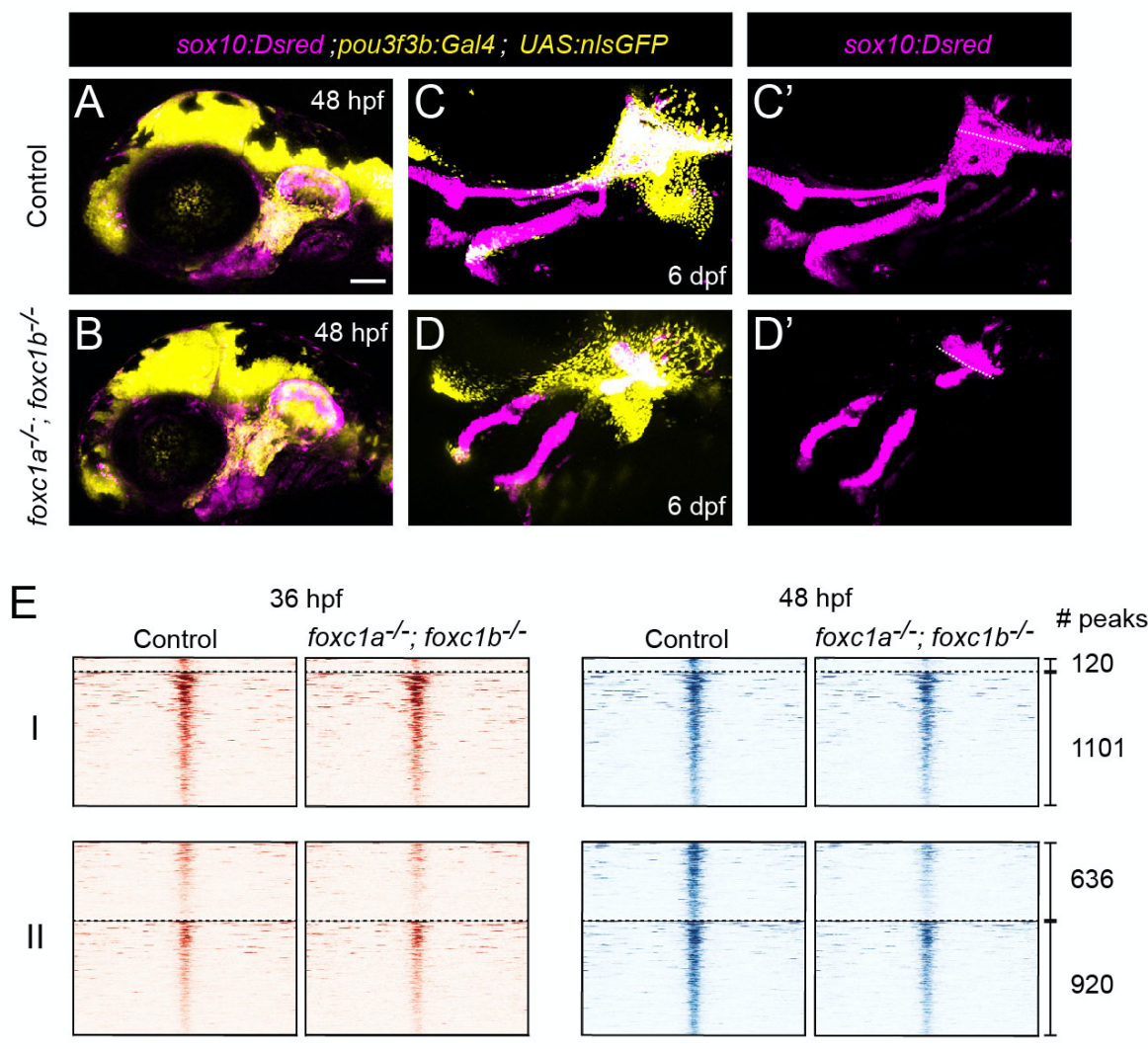

F

Group I elements

G Group II elements

Foxc1-dependent Foxc1-independent

Foxc1-dependent

Foxc1-independent
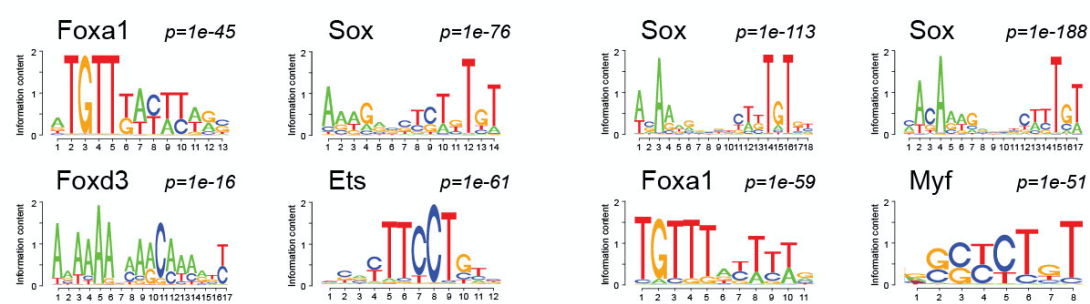

Foxa1 $p=1 e-59$
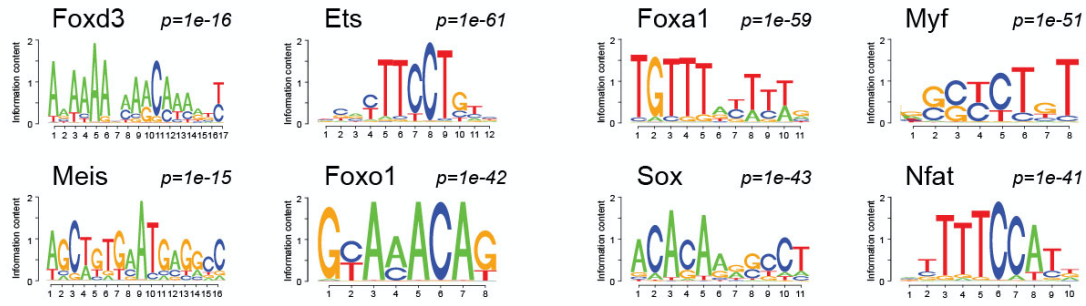

Sox $\quad p=1 e-43$
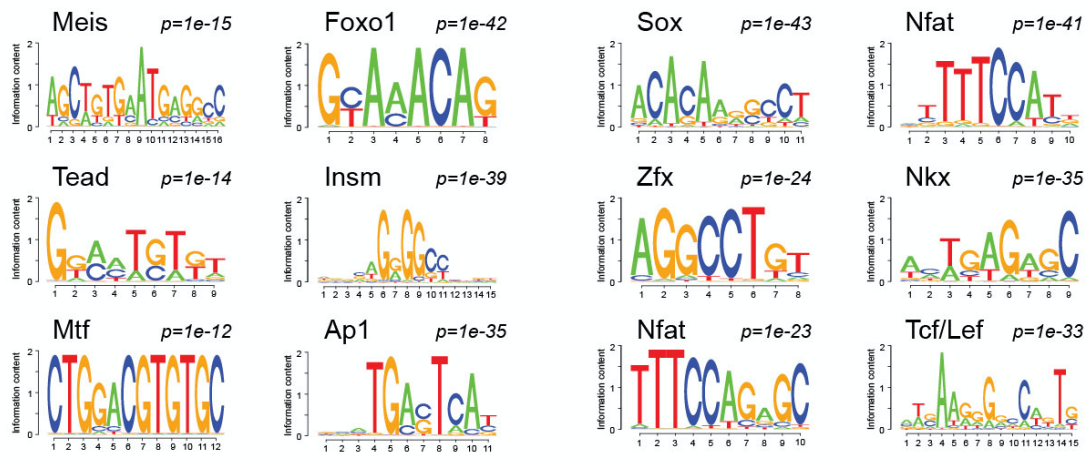

Nfat $\quad p=1 e-23$

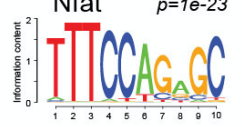

Tcf/Lef $\quad p=1 e-33$

Sox

$p=1 e-12$

Nfat $\quad p=1 e-33$

RunX $\quad p=1 e-23$
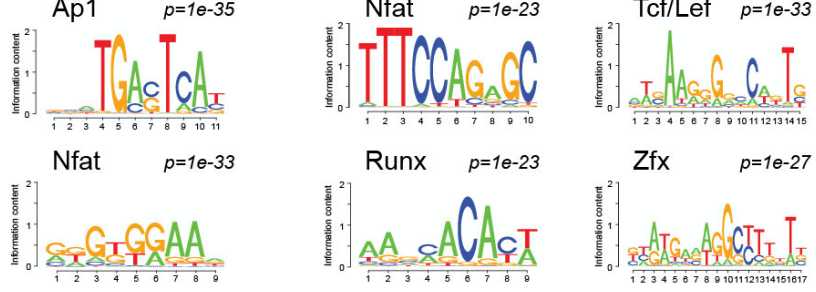
453 (A, B) Confocal images show dorsal CNCCs of the first two arches labeled by sox10:Dsred and

454 pou3f3b:Gal4; UAS:n/sGFP in control and foxc1 $\mathrm{a}^{-/-}$; foxc $1 \mathrm{~b}^{-/-}$mutant embryos at 48 hpf. Scale

455 bar $=100 \mu \mathrm{m} .(\mathrm{C}, \mathrm{D})$ Confocal images show loss of dorsal cartilages in foxc1 $a^{-/-}$; foxc $1 b^{-/-}$mutant

456 embryos at 6 dpf. sox10:Dsred+cartilages are seen in single channels in C' and D', with dashed

457 lines highlighting boundaries of dorsal and otic cartilage. (E) Peak intensity plots of Group I and

458 Group II elements in control and foxc1 $a^{---}$; foxc $1 b^{-/-}$mutant embryos. Peaks above the dashed

459 lines are reduced in mutants. $(F, G)$ De novo motif enrichment of Foxc1-dependent and Foxc1-

460 independent Group I and Group II elements. Top 6 motifs are shown with associated P values

461 after removing redundant motifs. 
bioRxiv preprint doi: https://doi.org/10.1101/2020.10.15.340703; this version posted October 15,2020 . The copyright holder for this preprint (which was not certified by peer review) is the author/funder, who has granted bioRxiv a license to display the preprint in perpetuity. It is made available under aCC-BY 4.0 International license.

A

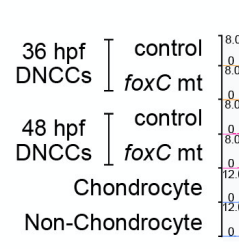
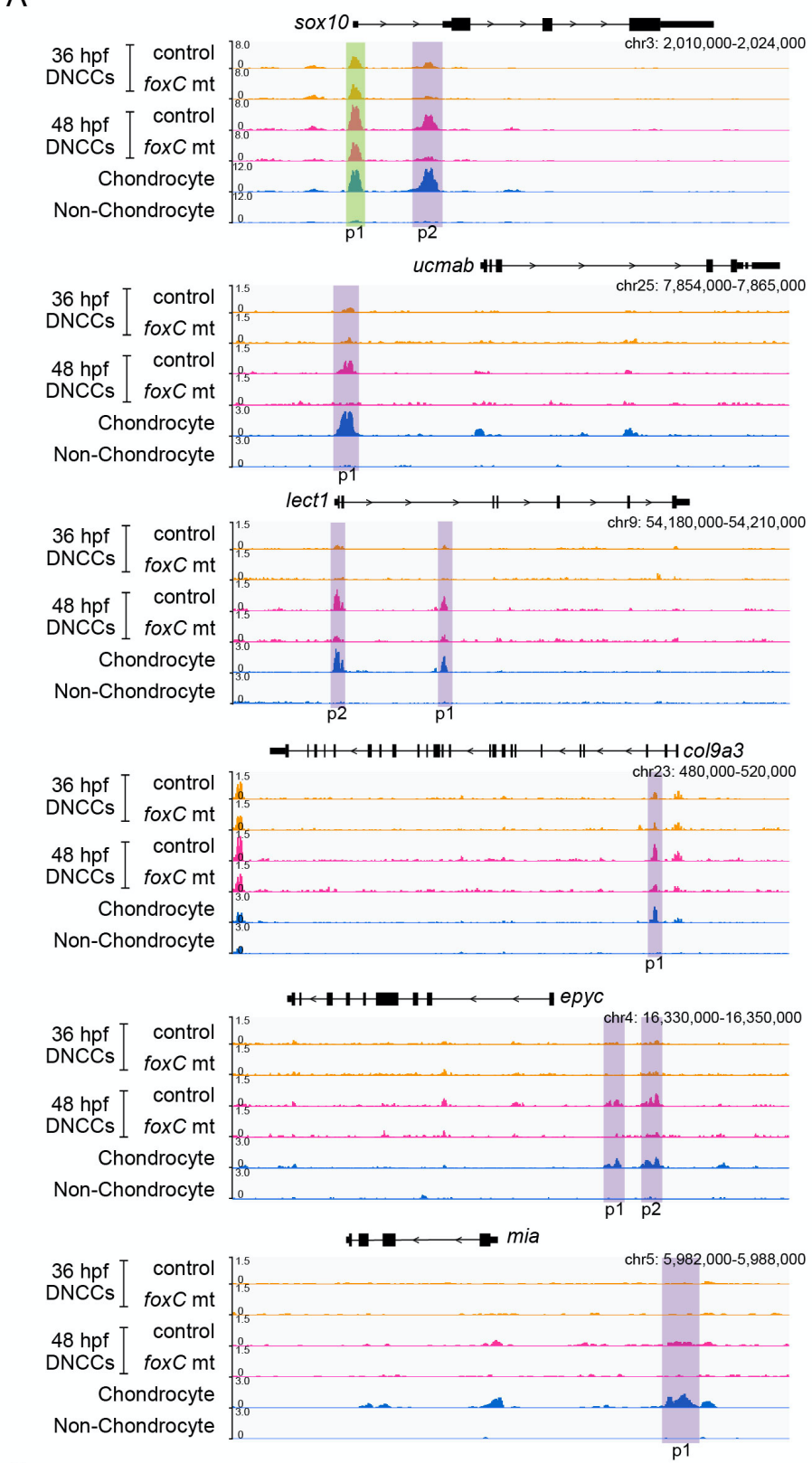

C
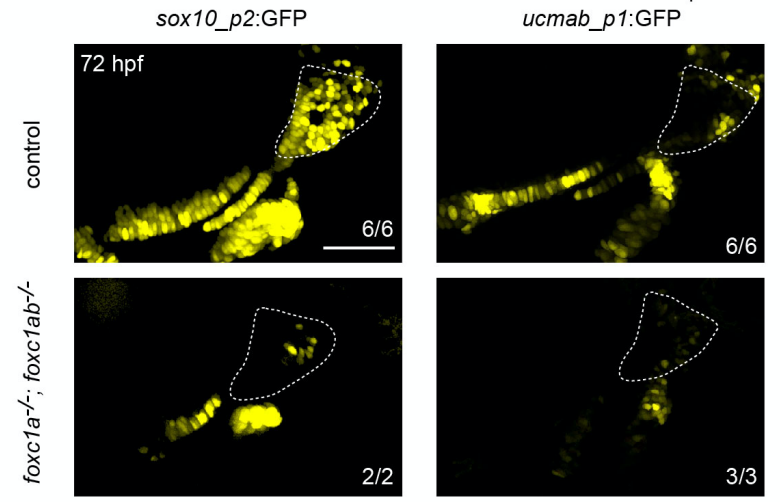

B
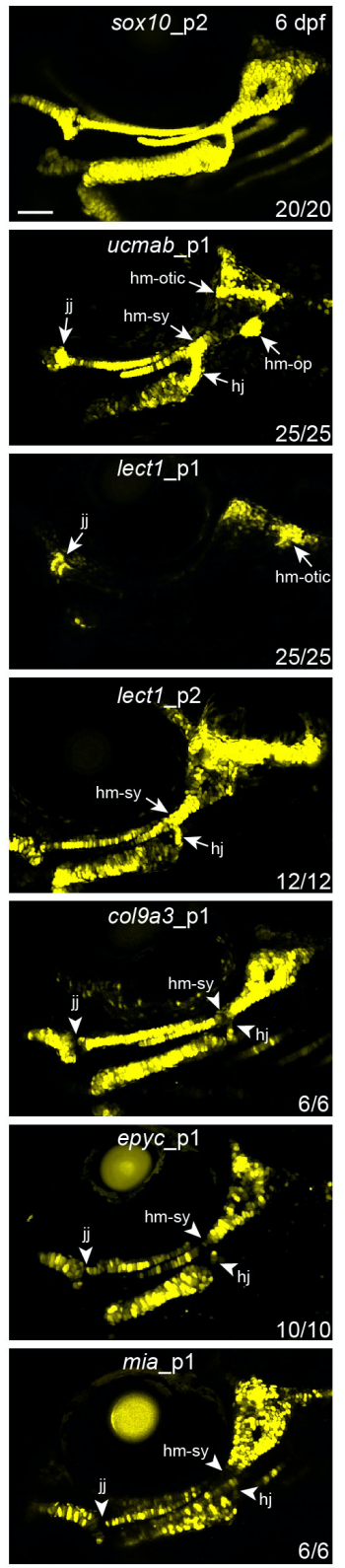

epyc_p1:GFP
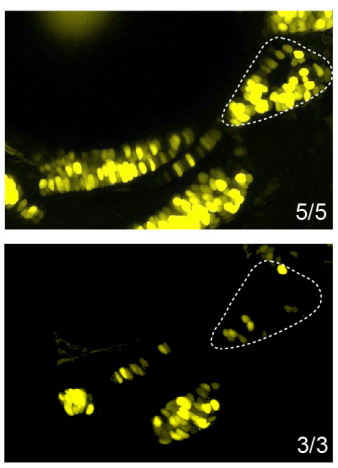


\section{Figure 4. In vivo validation of Foxc1-dependent cartilage enhancers}

465 (A) Snapshots of genomic regions (genes and GRCz10 coordinates listed) for enhancer testing.

466 Peaks (p) tested are shown, with Foxc1-dependent regions in purple and a Foxc1-independent

467 element in green. $\mu$ ATACseq reads are shown in each row for the experiments indicated, with

468 chondrocyte and non-chondrocyte peaks from 72 hpf embryos. DNCCs, dorsal CNCCs. (B)

469 GFP expression driven by the indicated peaks in stable transgenic zebrafish at 6 dpf. Confocal

470 projections of the cartilages of the first two arches are shown in lateral view with anterior to the

471 left. Arrows indicate enriched expression at joint regions, and arrowheads denote relative lack of

472 expression. jj, jaw joint. hj, hyoid joint. hm-sy, hyomandibular-symplectic junction. hm-otic,

473 hyomandibular-otic junction. hm-op, hyomandibular-opercular joint. (C) Confocal projections

474 show selective loss of sox10_p2:EGFP, ucmab_p1:EGFP, and epyc_p1:EGFP transgene

475 expression in the dorsal cartilage domains (dashed outlines) of foxc $1 a^{-/-}$; foxc $1 b^{-/-}$mutants at 72

476 hpf. Numbers indicate proportion of embryos in which the displayed patterns were observed.

477 Scale bars $=100 \mu \mathrm{m}$. 
Figure 1-figure supplement 1. col2a1a enhancer and cartilage motif comparison between

zebrafish and mouse

483 (A) Snapshot of the col2a1a locus showing MATACseq reads from the indicated experiments.

484 Several regions become accessible in $48 \mathrm{hpf}$ CNCCs and then remain open in chondrocytes at

48572 hpf. Boxed region shows the published R2 cartilage enhancer. (B) Comparison of consensus

486 motif sequences recovered from zebrafish $\mu$ ATACseq peaks enriched in facial chondrocytes

487 (left) and mouse Sox9 ChIP-Seq peaks in rib chondrocytes (right).

489 Figure 3-figure supplement 1. Comparison of accessible regions between pan- and

490 dorsal CNCCs

491 Venn diagrams and heatmaps show distal accessible elements in $\mu$ ATACseq data from

492 fli1a:GFP+; sox10:Dsred+ (pan-CNCCs) and pou3f3b>GFP+; sox10:DsRed+ (dorsal CNCCs) at

$49336 \mathrm{hpf}(\mathrm{A})$ and $48 \mathrm{hpf}(\mathrm{B})$. Numbers list elements unique for each population or shared (center of

494 circles). 
496 Figure 3-figure supplement 2. Accessibility of cartilage-enriched elements in earlier pan-

497 and dorsal CNCCs.

$498(A, B)$ Heatmaps of the 5,736 distal elements with accessibility in 72 hpf chondrocytes. Their

499 accessibility is then plotted according to $\mu A T A C s e q$ data from fli1a:GFP+; sox10:Dsred+ (pan-

500 CNCCs) and pou3f3b>GFP+; sox10:Dsred+ (dorsal CNCCs) at 36 hpf (A) and 48 hpf (B). At 36

501 hpf, 132 peaks were more accessible in pan-CNCCs and 106 peaks more accessible in dorsal

502 CNCCs. At 48 hpf, 2,470 peaks were more accessible in pan-CNCCs and 10 peaks more

503 accessible in dorsal CNCCs. Peaks commonly or not accessible in both data sets are also

504 shown. (C,D) Pearson correlation graphs of the behavior of cartilage-associated distal elements

505 in pan- versus dorsal CNCCs at 36 and $48 \mathrm{hpf} . \mathrm{x}$ and y axes represent raw read counts from

506 BAM files, and "r" is the Pearson correlation coefficient.

508 Figure 4-figure supplement 1. Additional in vivo validation of cartilage-enriched

509 accessible elements

510 (A) Schematic of the reporter construct for enhancer testing. Tol2, transposase integration site.

$511 \mathrm{E} 1 \mathrm{~b}$, minimal promoter. GFP, green fluorescent protein. pA, polyadenylation sequence. CFP, 
512 cerulean fluorescent protein. (B) Snapshots of genomic regions (genes and GRCz10

513 coordinates listed) for enhancer testing. Peaks ( $p)$ tested are shown, with Foxc1-dependent

514 regions in purple and Foxc1-independent elements in green. $\mu A T A C s e q$ reads are shown in

515 each row for the experiments indicated, with chondrocyte and non-chondrocyte peaks from 72

516 hpf embryos. DNCCs, dorsal CNCCs. (C) GFP expression (yellow) driven by the indicated

517 peaks in stable transgenic zebrafish at $6 \mathrm{dpf}$. Confocal projections of the cartilages of the first

518 two arches are shown in lateral view for acan_p1 and ventral view for col9a1a_p1. For acan_p1,

519 sox10:Dsred labels all chondrocytes for reference in magenta, and arrow indicates hyoid joint

520 expression. For col9a1a_p1, arrows indicate expression in the ventral Meckel's and ceratohyal

521 cartilages. (D) Confocal sections of representative embryos injected with enhancer constructs

522 are shown in lateral view at $6 \mathrm{dpf}$. Mosaic expression in chondrocytes (yellow, arrows) is shown

523 with DIC providing embryo context in white. For sparc_p1, interopercular ligament expression is

524 denoted by arrowhead. Numbers indicate proportion of embryos in which the displayed patterns

525 were observed. Scale bar $=100 \mu \mathrm{m}$. 
A
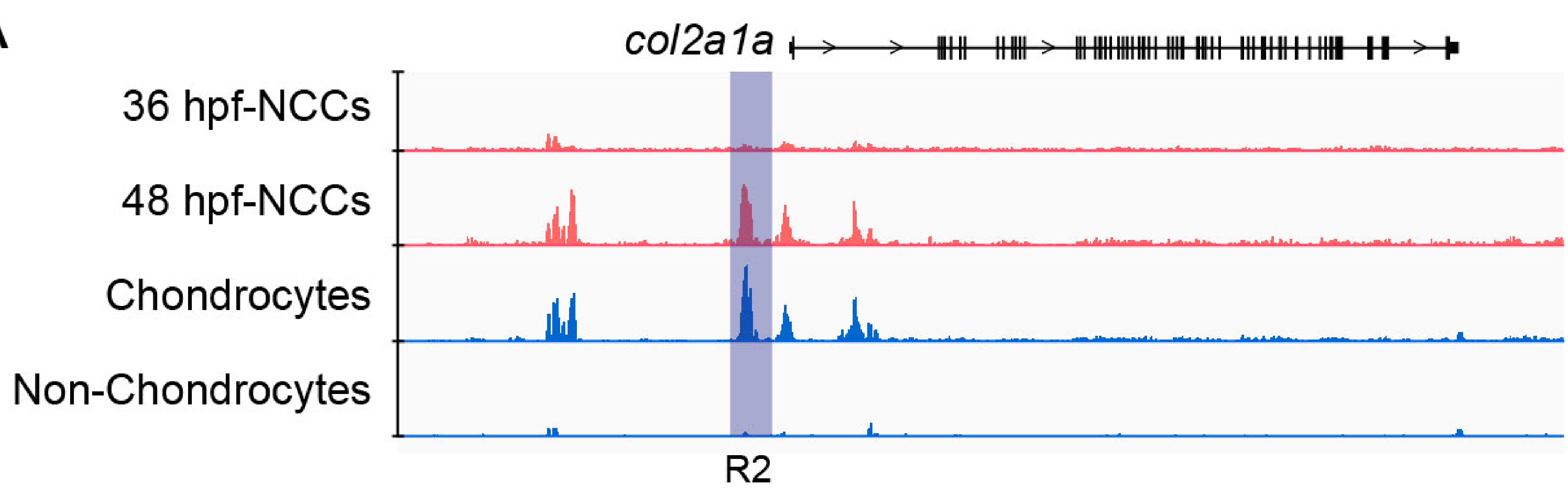

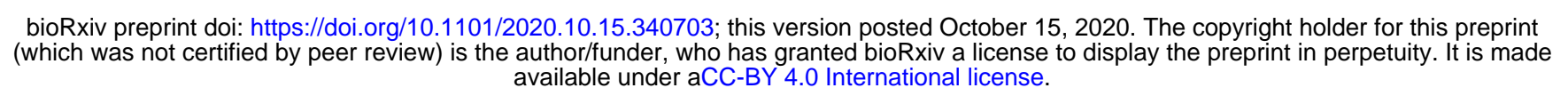

B

\begin{tabular}{|c|c|}
\hline Zebrafish motif sequences & $\begin{array}{l}\text { Mouse motif sequences } \\
\text { (Ohba et al., 2015) }\end{array}$ \\
\hline Sox & Sox \\
\hline Fox & Fox \\
\hline Nfat & Nfat \\
\hline
\end{tabular}


A

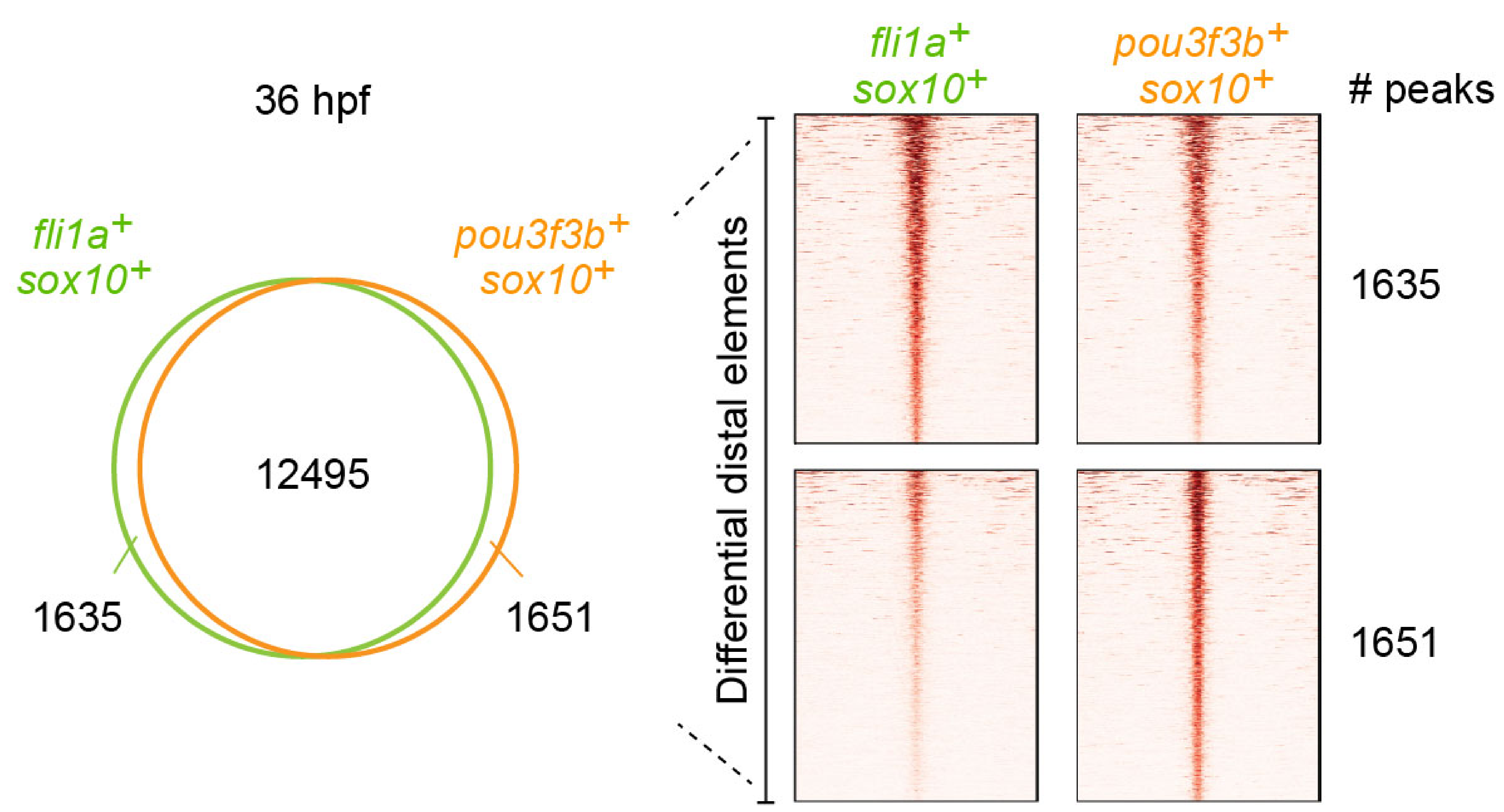

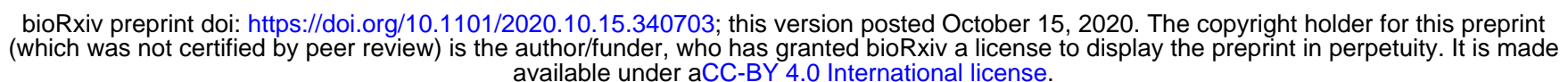

B

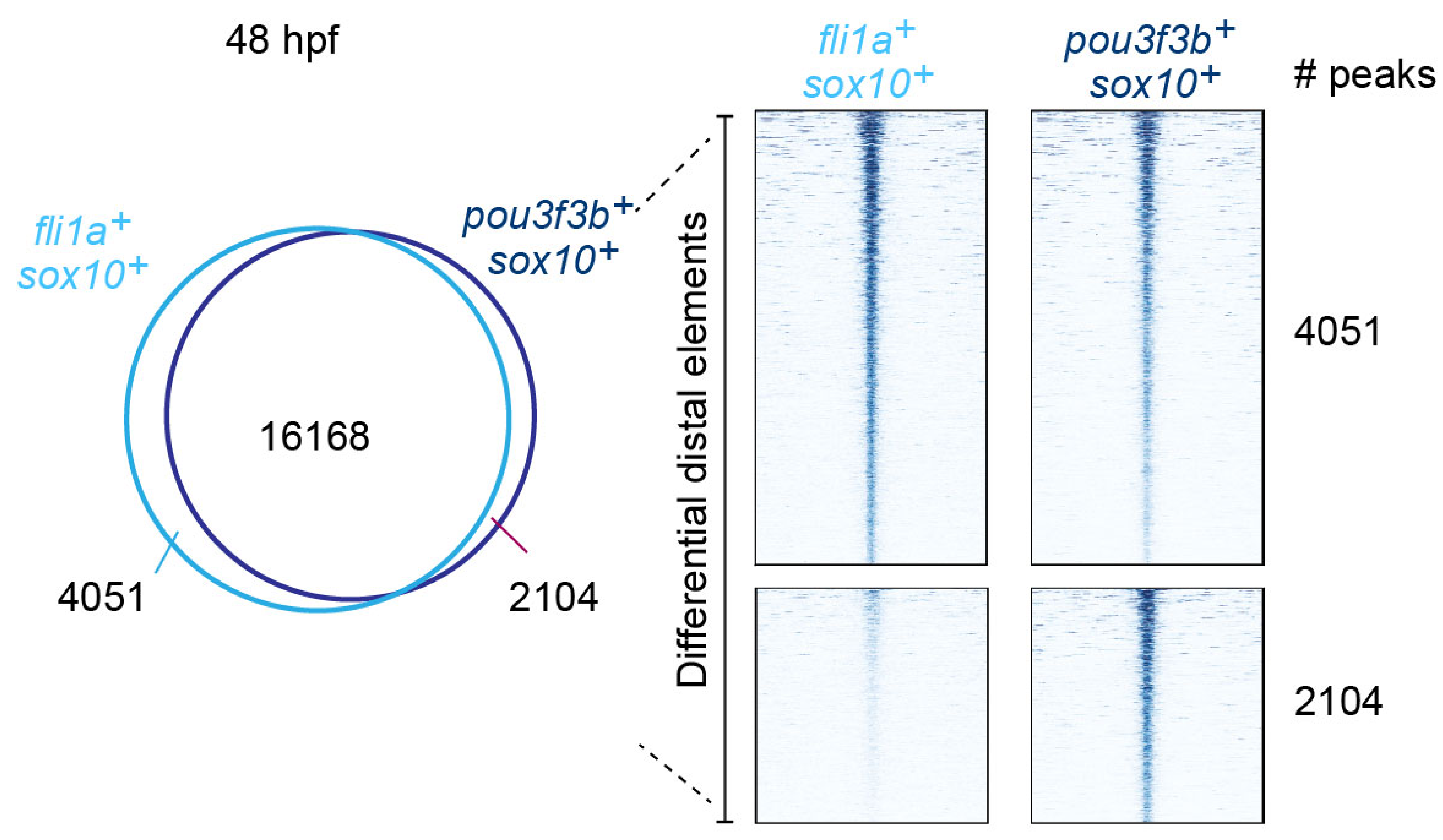


A

$36 \mathrm{hpf}$

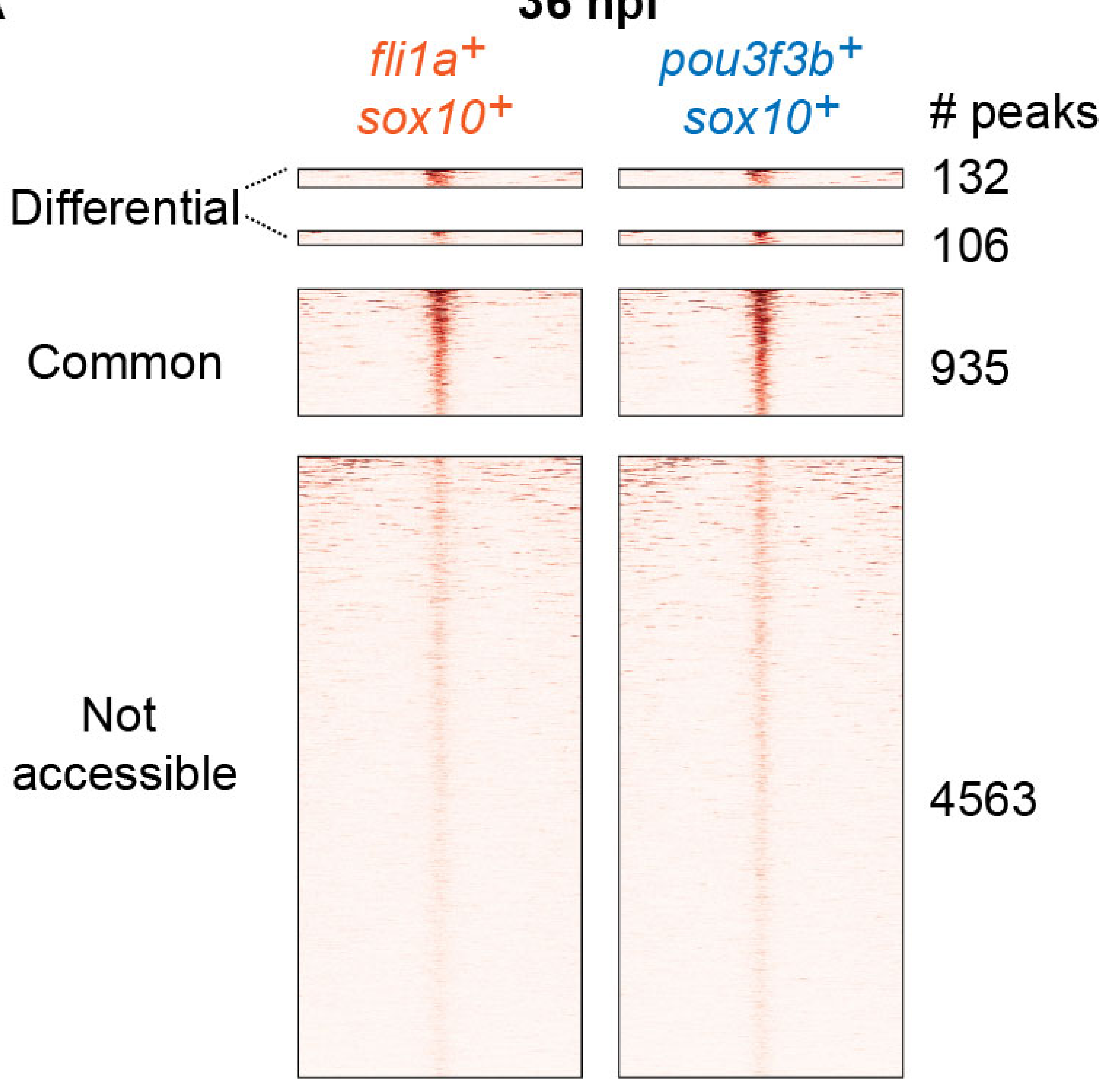

C

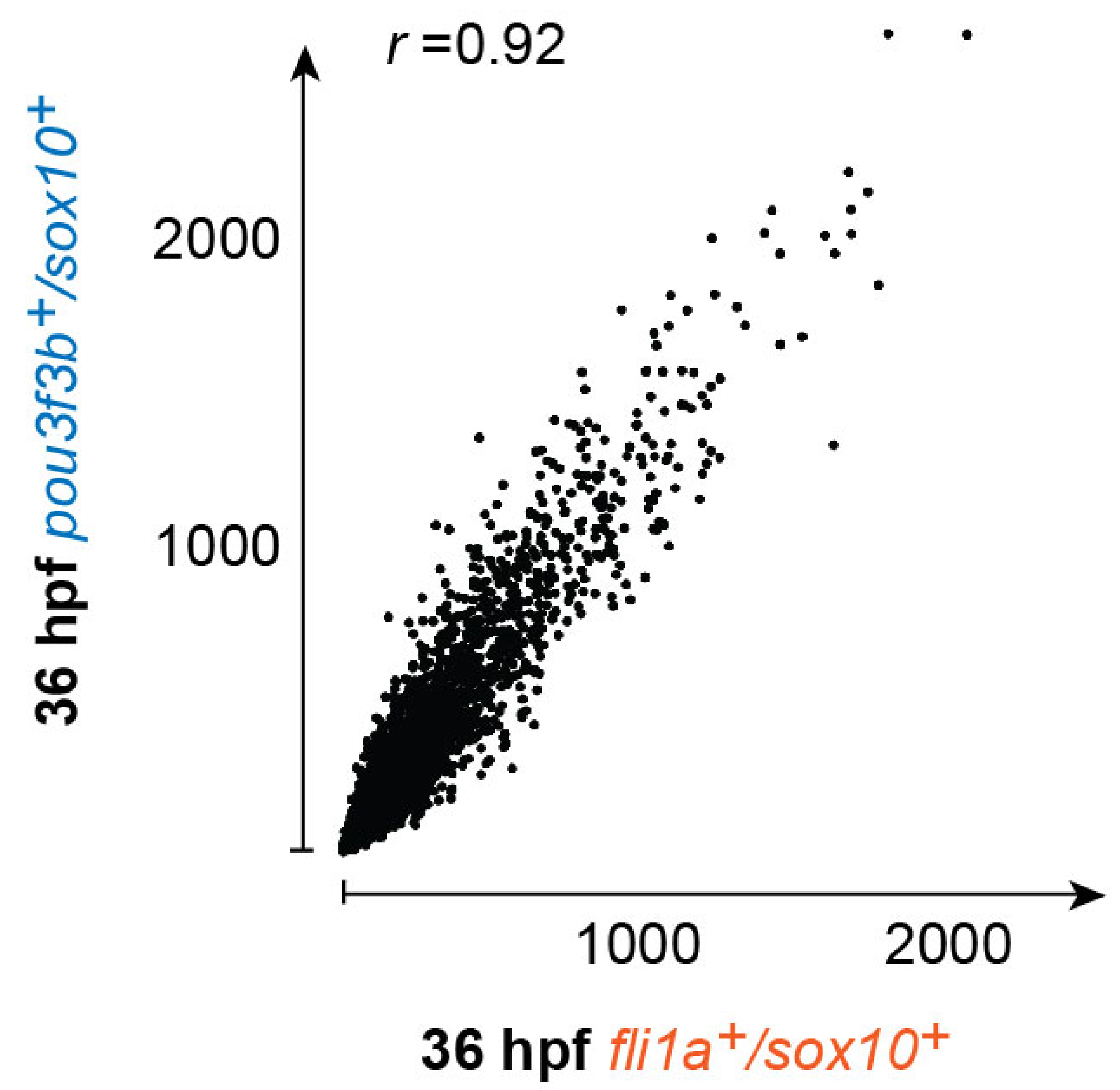

B

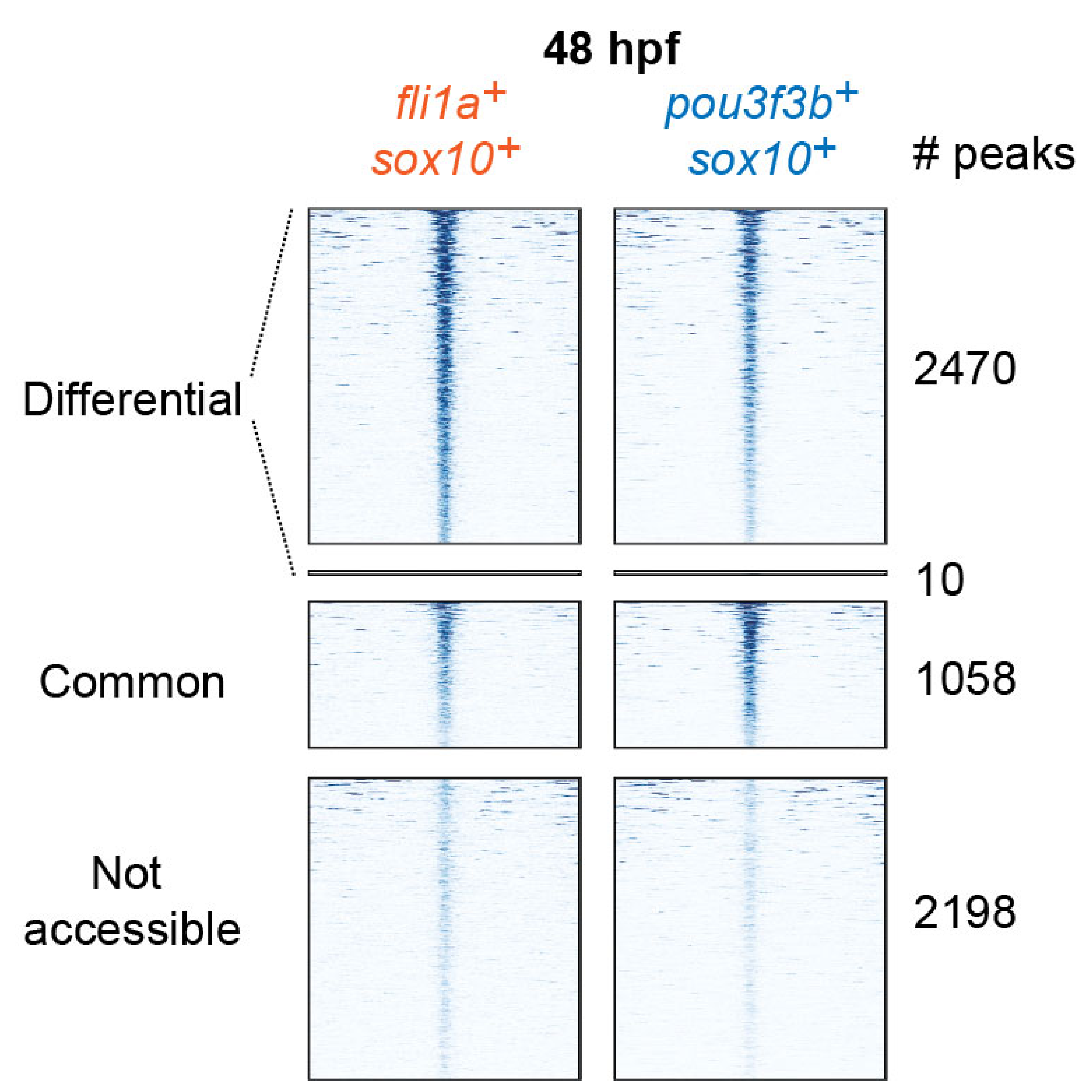

D

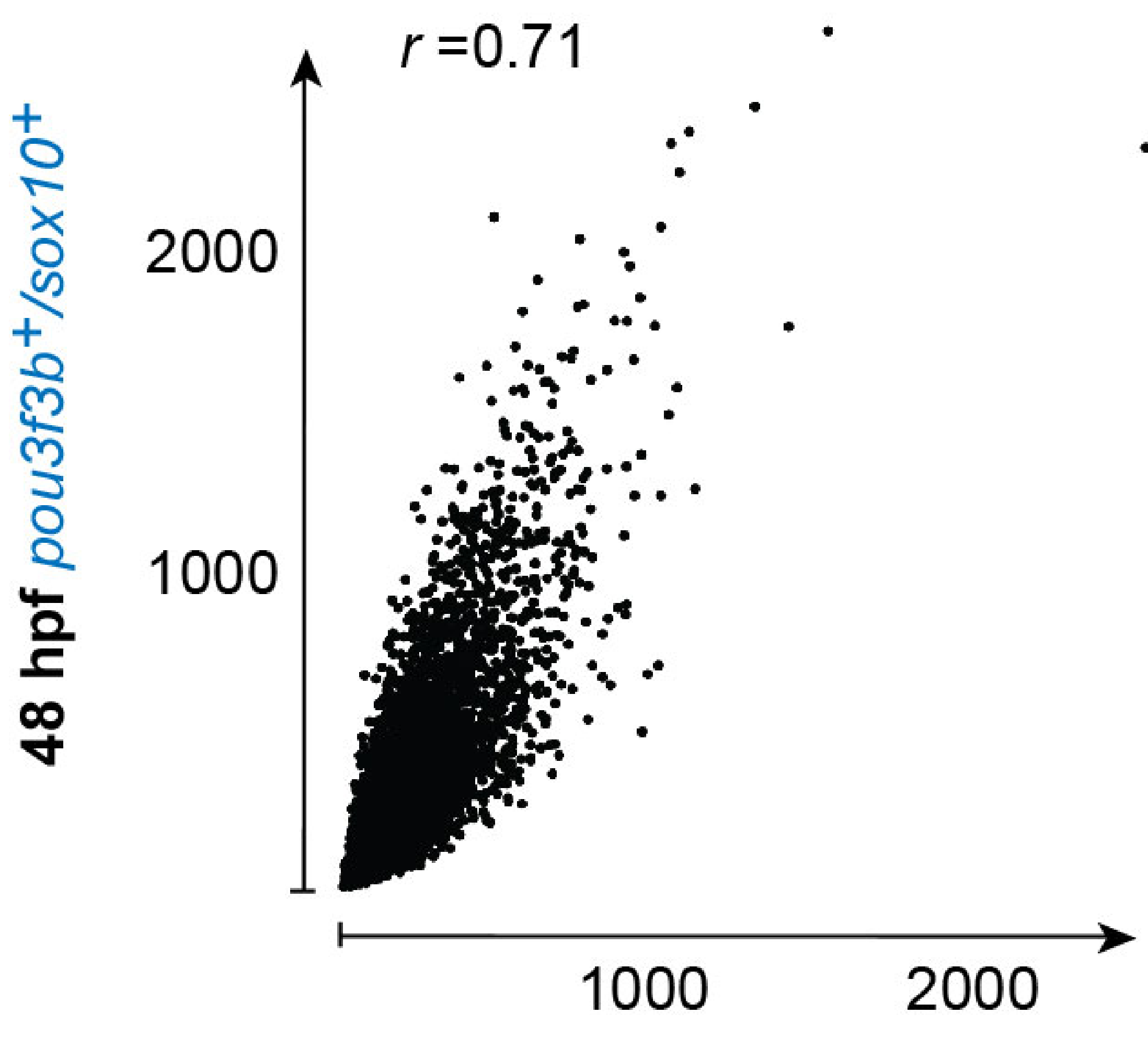

48 hpf $\mathrm{fli}^{+} \mathrm{a}^{+} / \mathrm{so} \times 10^{+}$ 
A

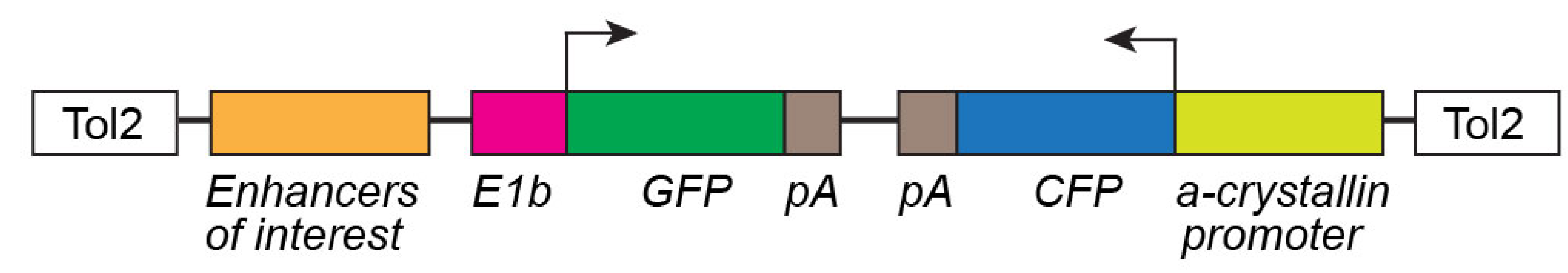

B
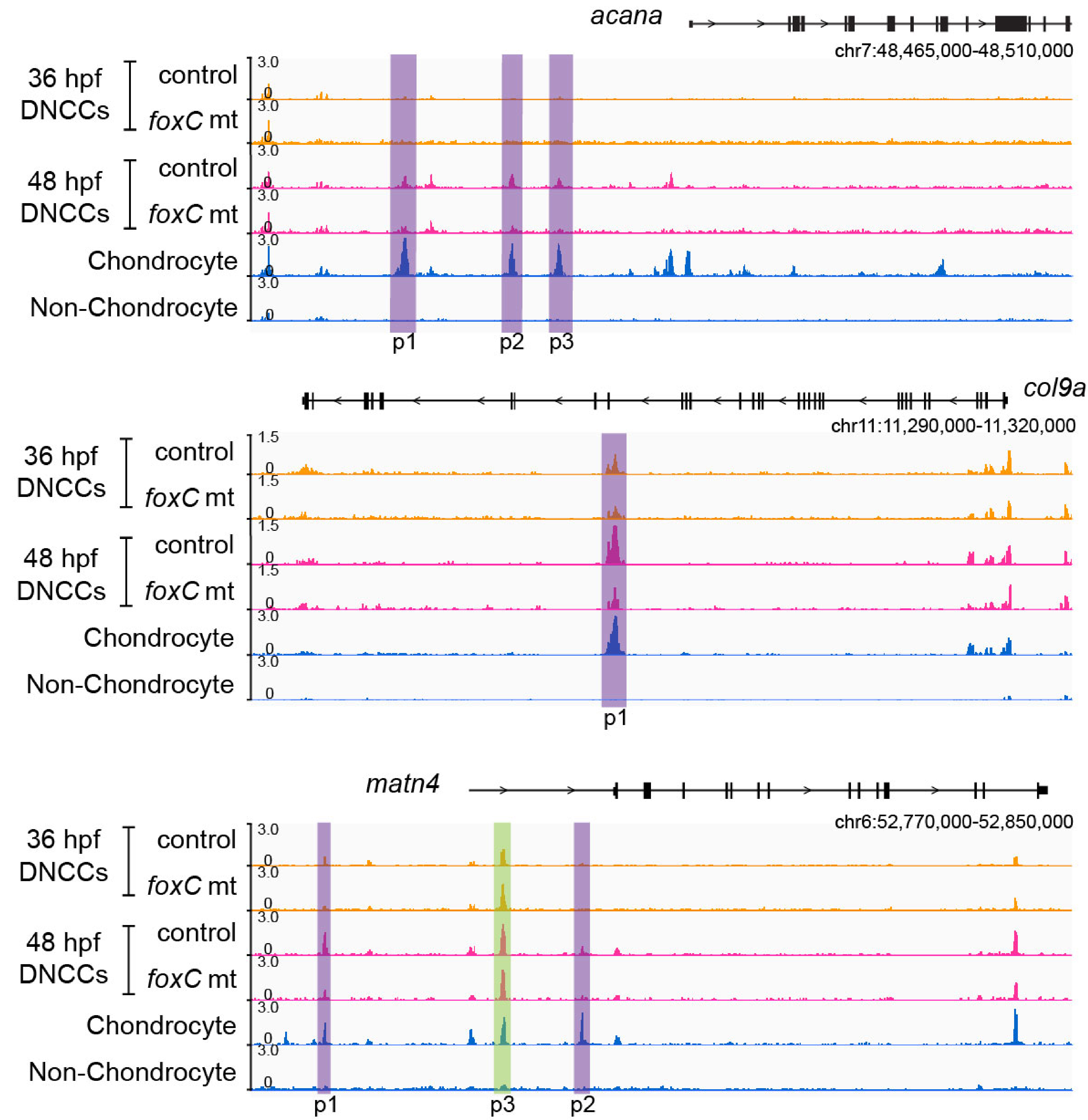

gas $1 b$
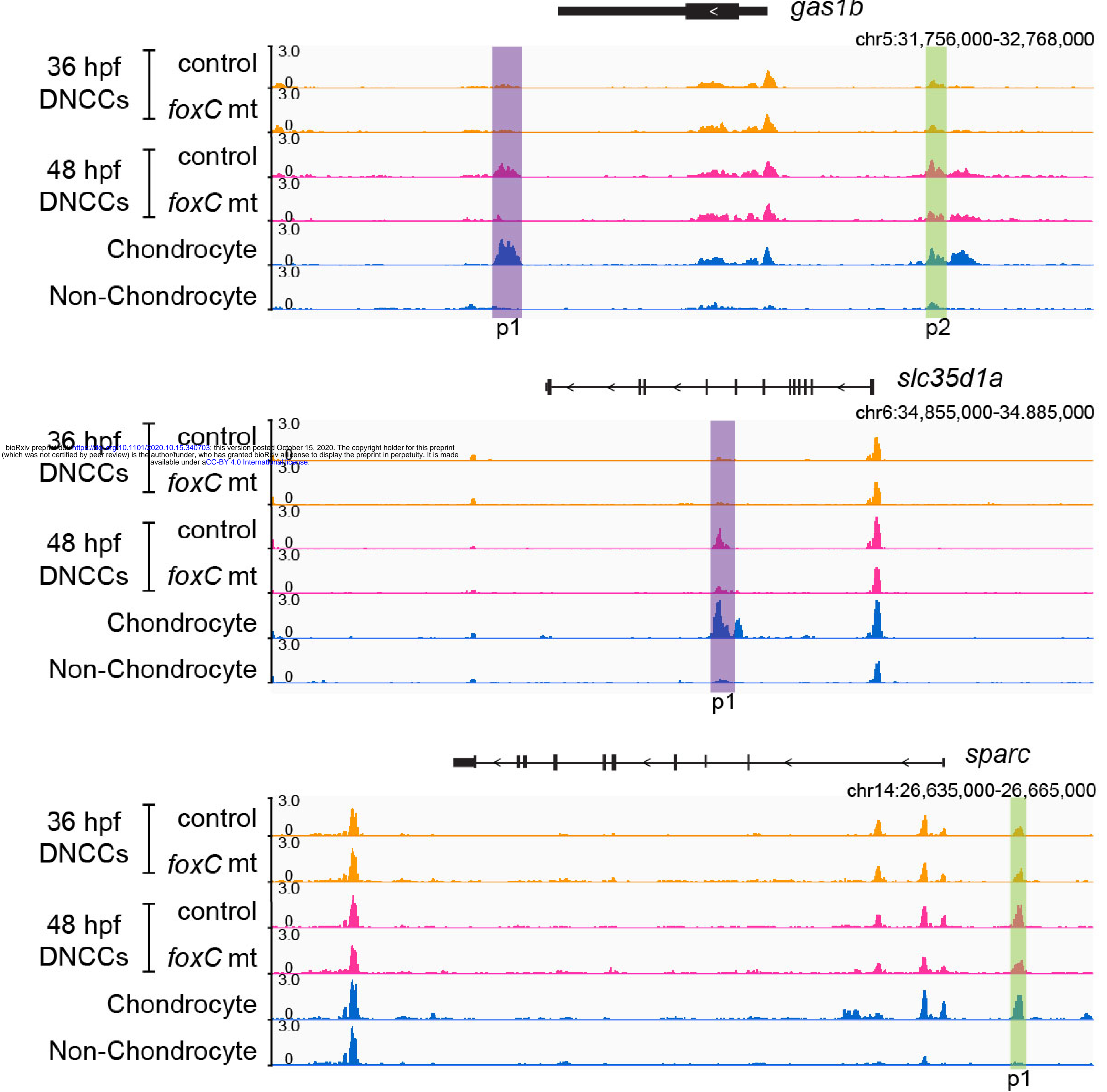

C

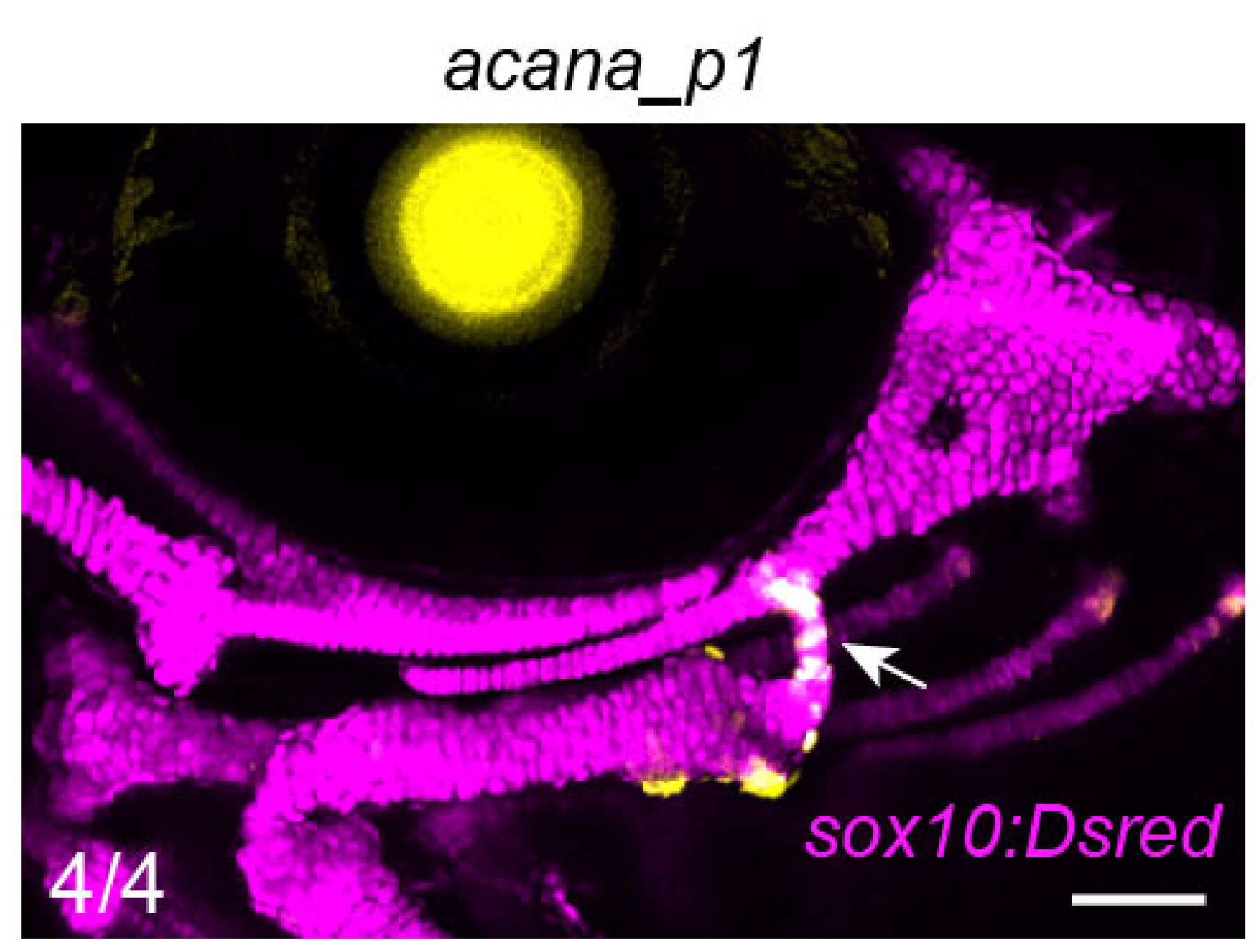

col9a1a_p1

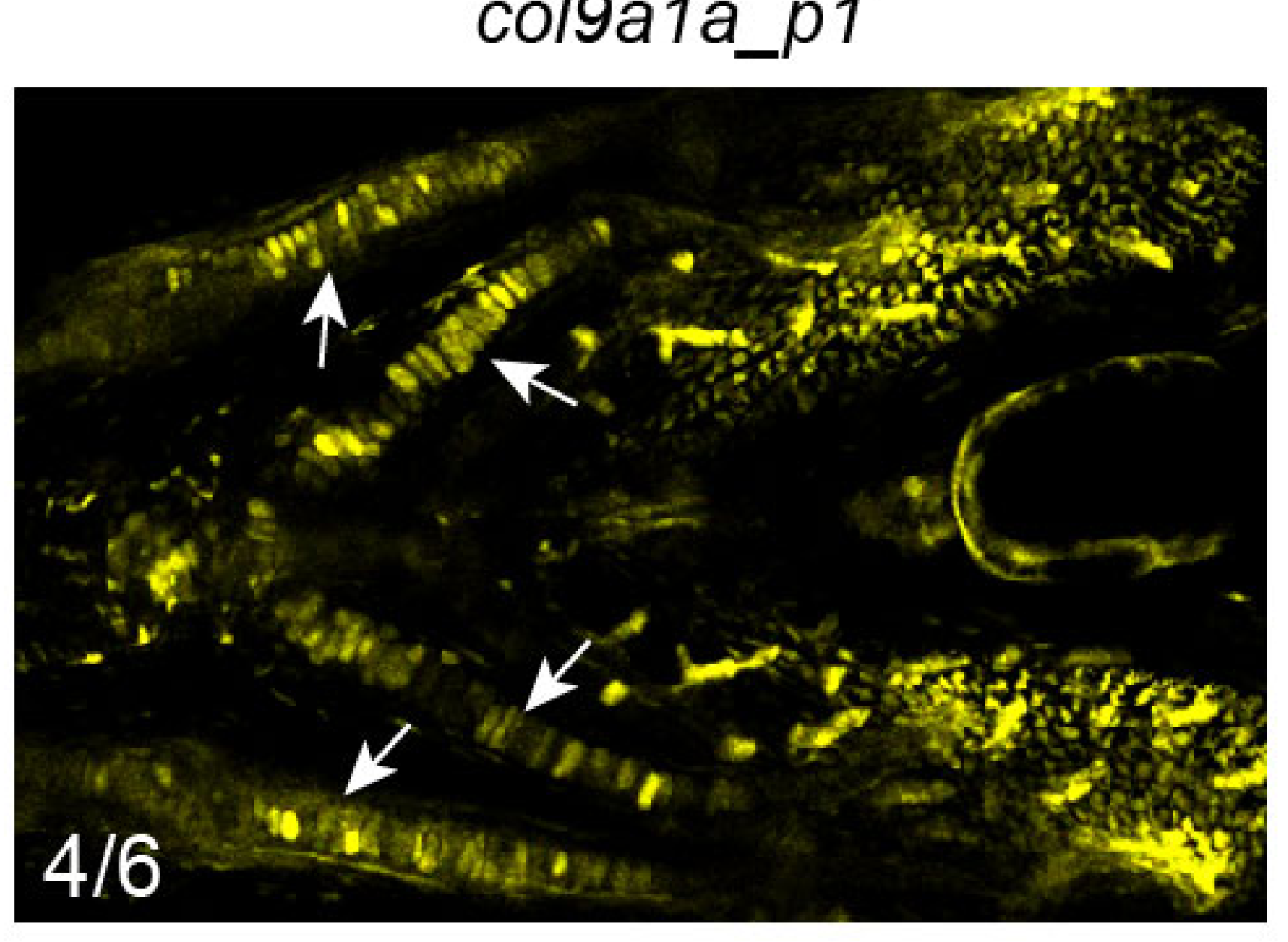

D

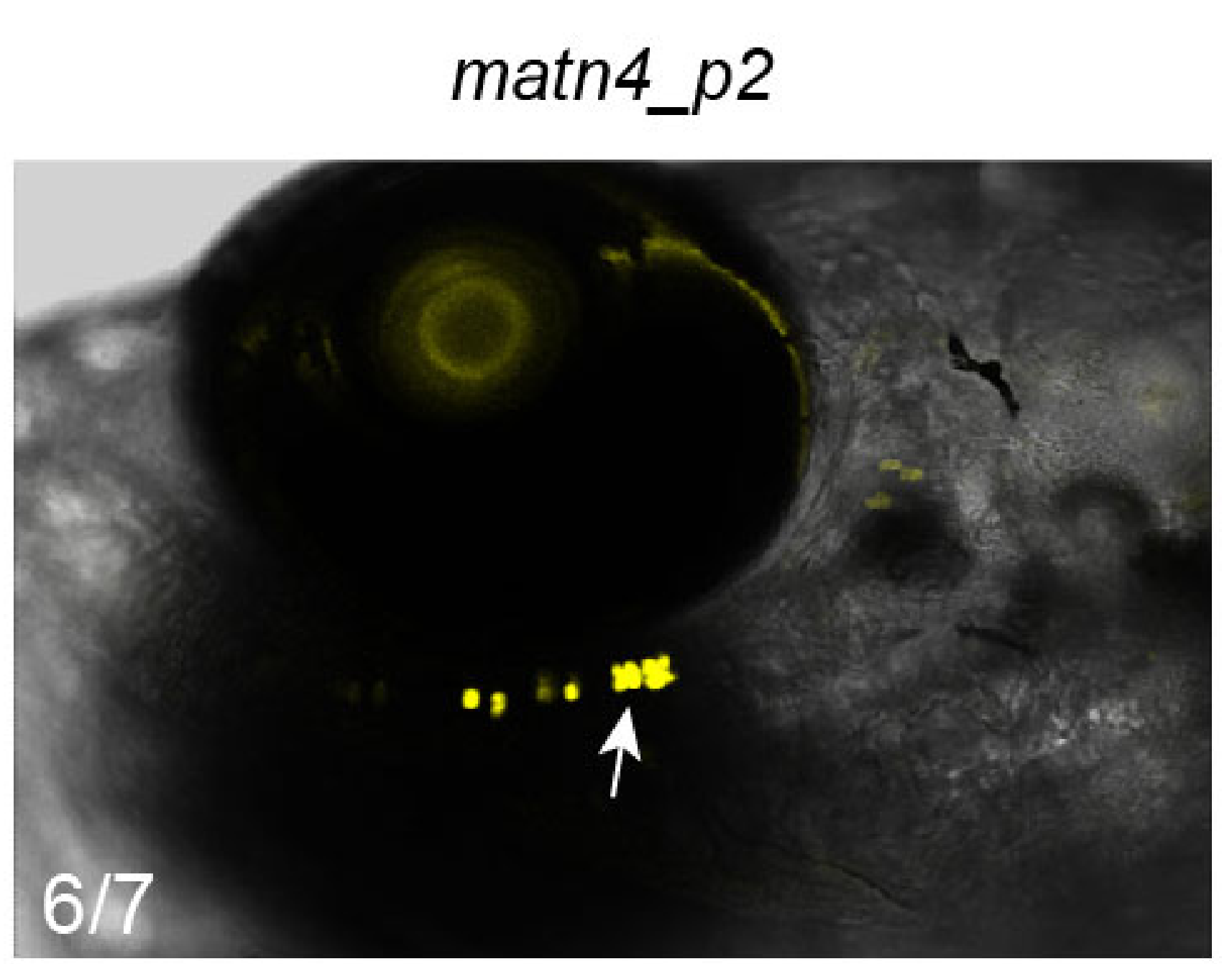

gas1b_p1

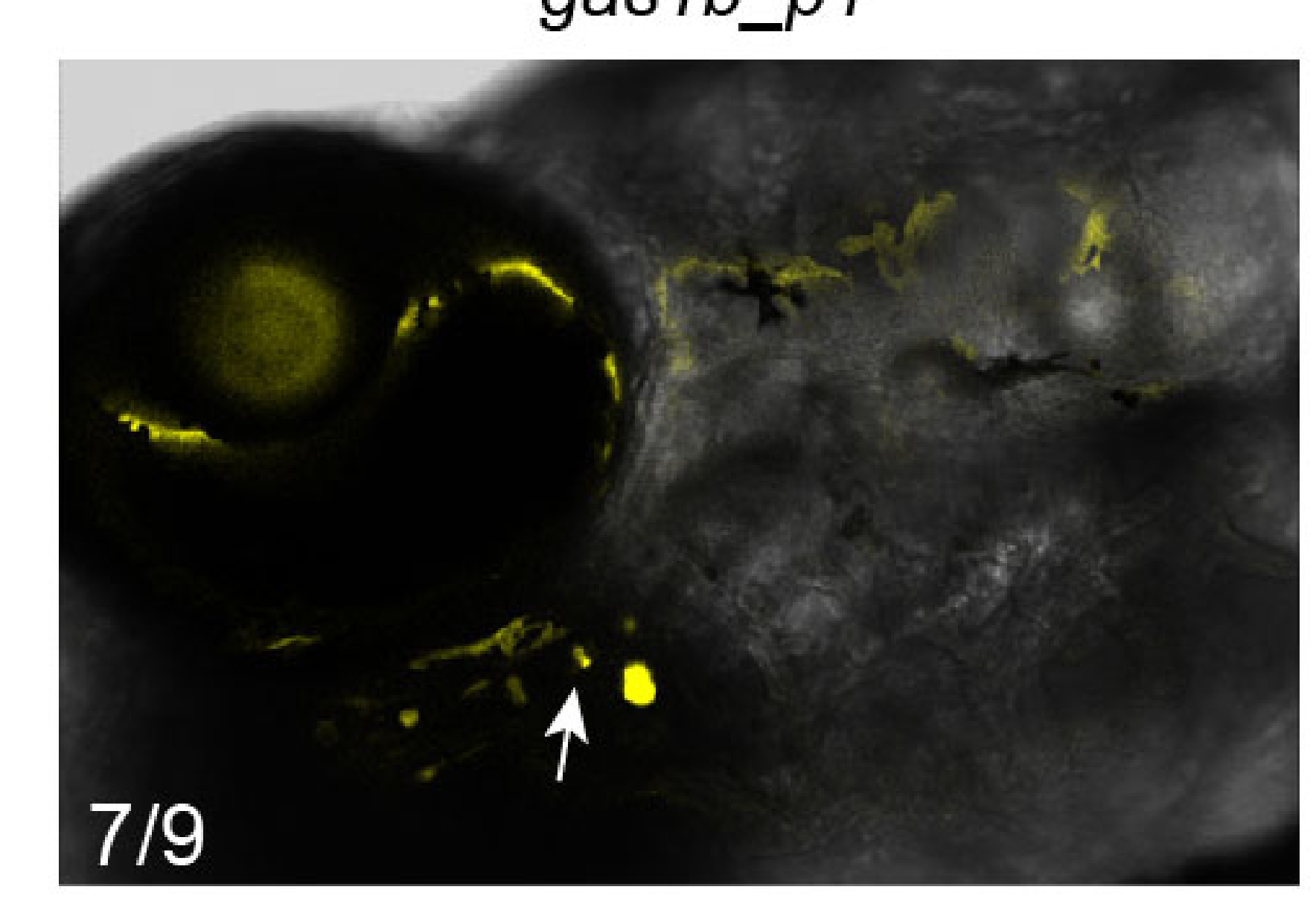

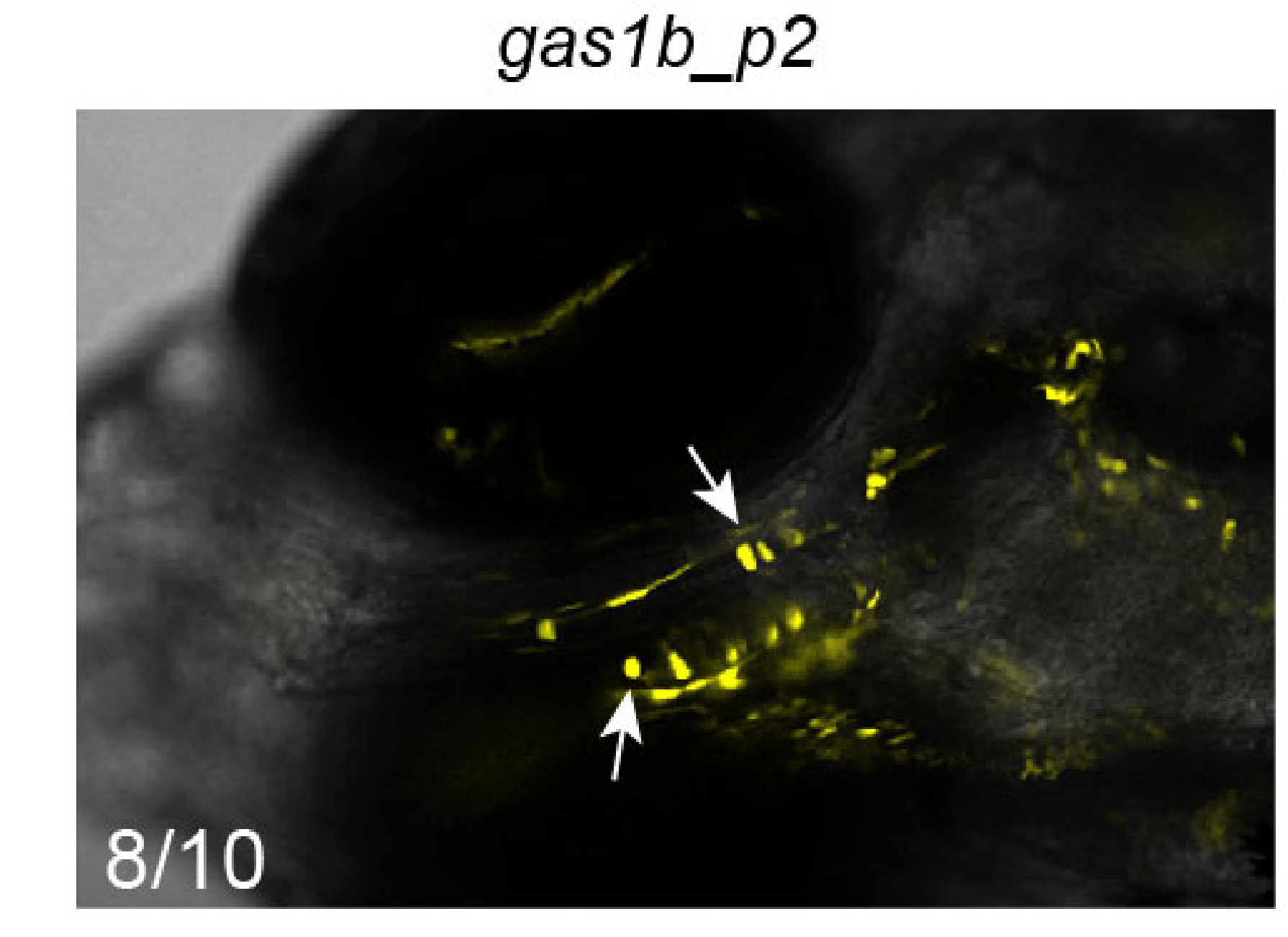

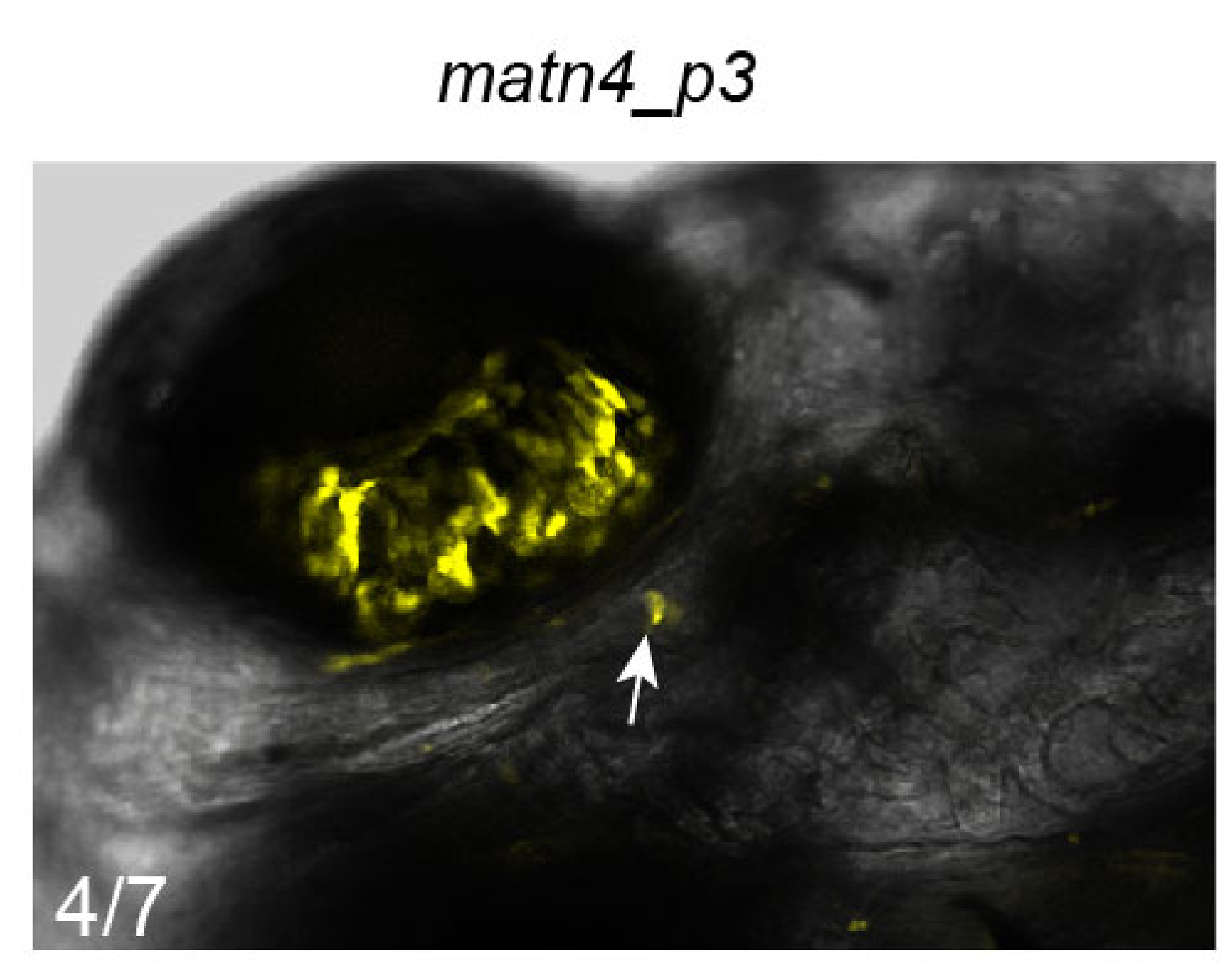

gas1b_p2
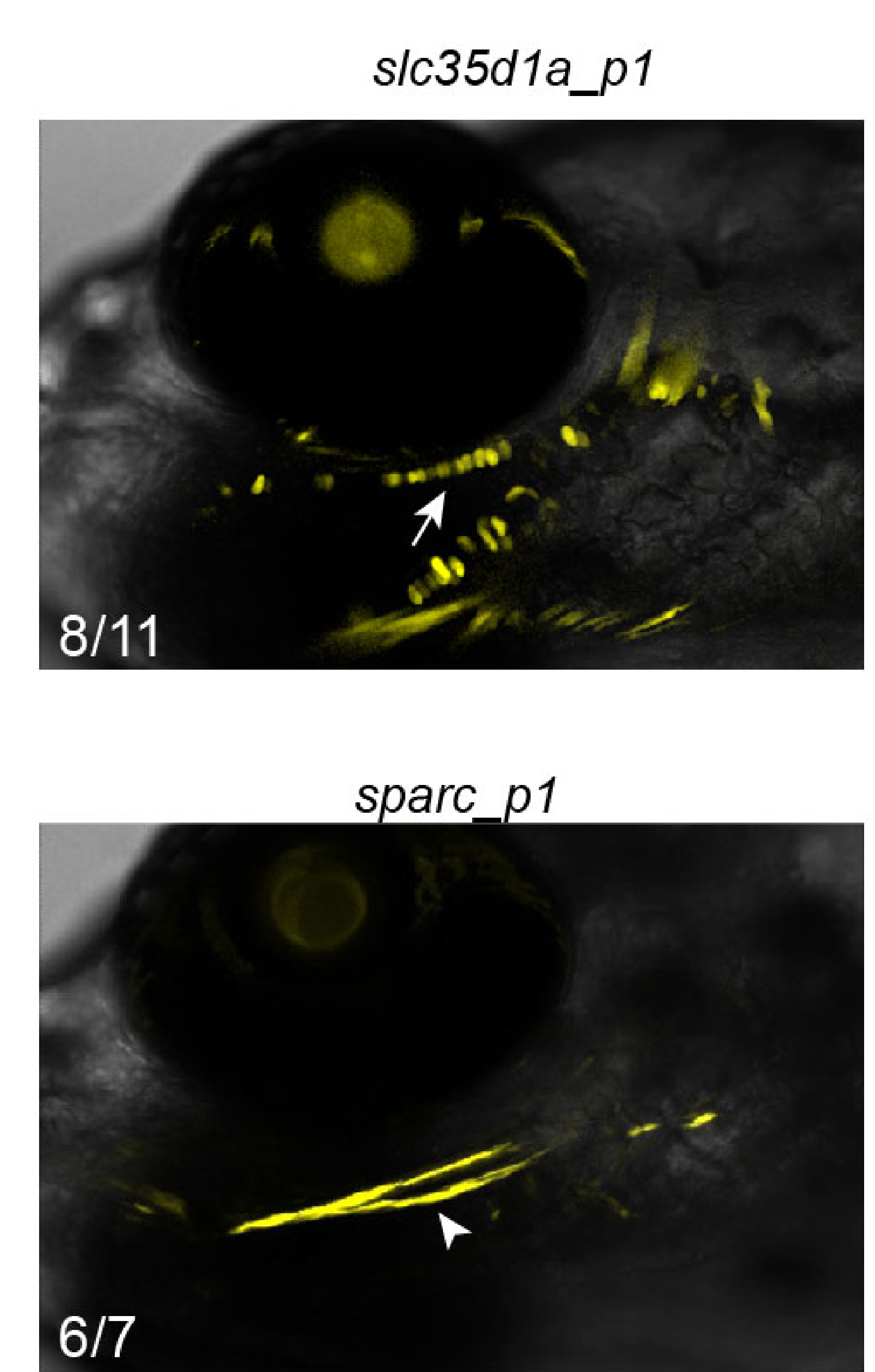